\title{
Search for cold debris disks around M-dwarfs. II
}

\author{
J.-F. Lestrade ${ }^{1}$, M. C. Wyatt ${ }^{2}$, F. Bertoldi ${ }^{3}$, K. M. Menten ${ }^{4}$, and G. Labaigt ${ }^{5}$ \\ 1 Observatoire de Paris - CNRS, 77 Av. Denfert Rochereau, 75014 Paris, France \\ e-mail: jean-francois. lestrade@obspm.fr \\ 2 Institute of Astronomy, University of Cambridge, Cambridge, CB3 OHA, UK \\ e-mail:wyatt@ast.cam.ac.uk \\ 3 Argelander Institute for Astronomy, University of Bonn, Auf dem Hügel 71, 53121 Bonn, Germany \\ e-mail: bertoldi@astro.uni-bonn.de \\ 4 Max-Planck-Institut für Radioastronomie, Auf dem Hügel 69, 53121 Bonn, Germany \\ e-mail: kmenten@mpifr-bonn.mpg.de \\ 5 École Normale Supérieure de Cachan, 61 avenue du Président Wilson, 94235 Cachan, France \\ e-mail: glabaigt@rip.ens-cachan.fr
}

Received 8 April 2009 / Accepted 6 July 2009

\section{ABSTRACT}

\begin{abstract}
Although $70 \%$ of the stars in the Galaxy are M-dwarfs, thermal emission searches for cold debris disks have been conducted mostly for A-type and solar-type stars. We report on new $\lambda=1.2 \mathrm{~mm}$ continuum observations of thirty M-dwarfs, using the MAMBO-2 bolometer array camera at the IRAM $30 \mathrm{~m}$ telescope. For a statistical analysis, we combine these data with our prior SCUBA and MAMBO-2 observations of 20 other M-dwarfs. Our sample consists of M-dwarfs in moving groups, with relatively young ages, and of nearby M-dwarfs with unknown ages. Only one cold debris disk (GJ842.2) was detected significantly. We compare the implied disk abundance constraints with those found in two comparable submillimeter surveys of 10 to $190 \mathrm{Myr}$ old A- and FGK-type stars. For the 19 youngest (ages less than $200 \mathrm{Myr}$ ) M-dwarfs in our sample, we derive a cold disk fraction of $5.3_{-5.0}^{+10.5} \%$, compared to $15_{-11.5}^{+11.5} \%$ for FGK-stars and $22_{-20}^{+33} \%$ for A-stars. Hence, for this age group, there is an apparent trend of fewer cold disks for later stellar types. Although its statistical significance is marginal, this trend is strengthened by the deeper observations of our M-dwarf sample. We derive a cold disk fraction of $<10 \%$ for the older (likely a few Gyr) M-dwarfs in our sample. Finally, although inconclusively related to a debris disk, we present the complex millimeter structure found around the position of the M 1.5 dwarf GJ526 in our sample.
\end{abstract}

Key words. stars: circumstellar matter - stars: low-mass, brown dwarfs - planetary systems: formation

\section{Introduction}

Cold debris disks around main sequence stars are left-over planetesimals (comets) that could not agglomerate into larger planets during the initial phase of planet formation. They are assembled as a belt within the periphery of the system in a manner analogous to the Kuiper Belt. The study of debris disks, including warm disks such as the asteroid belt in our Solar System, advances our knowledge of the origin and evolution of planetary systems around other stars, in a similar way to the study of the Kuiper Belt. It is well recognised that its present-day structure and dynamics retain important information about the formation and evolution of the Solar System. For example, the low mass and expanded size of the present-day Kuiper Belt can be traced back to the outward migrations of the giant planets, which exchanged orbital energy with an initially more compact and more massive disk (Hahn \& Malhotra 1999; Tsiganis et al. 2005; Morbidelli et al. 2005; Gomes et al. 2005).

Our current understanding of debris disks was reviewed by Wyatt (2008). Mutual collisions between planetesimals in debris disks produce second-generation dust grains that are observable by means of their thermal emission or scattered light. Since the discovery of a first debris disk around the A0 main sequence star Vega by IRAS (Aumann et al. 1984), debris disks have been searched for photometrically with the infrared (IR) satellites IRAS, ISO, and Spitzer. In these observations, any flux excess above the photospheric level is interpreted as emission from warm $(50-100 \mathrm{~K})$ circumstellar dust.

By analyzing Spitzer observations, it was found that $32 \pm 5 \%$ of 160 A-dwarfs show a $70 \mu \mathrm{m}$ excess (Su et al. 2006), while only $16_{-2.9}^{+2.8} \%$ of 225 observed FG-dwarfs show excess emission (Bryden et al. 2006; Trilling et al. 2008). Submillimeter photometry of A-to-G type stars with no detectable IRAS excess do however show cold $(10-50 \mathrm{~K})$ dust emission around some of them (Wyatt et al. 2003; Najita \& Williams 2005). Imaging of scattered light with HST and of thermal continuum emission with SCUBA has measured disk radii between 50 and 150 AU (Smith \& Terrile 1984; Kalas et al. 2005; Holland et al. 1998; Greaves et al. 2005); 22 debris disks have been imaged to date $^{1}$. Azimuthal structures have been detected in a few of these disks and are thought to be caused by dust associated with planetesimals trapped in mean motion resonance with an orbiting planet (Wyatt 2003, 2006; Reche et al. 2008), or by dynamical perturbations from a distant stellar companion or passing stars (HD 141569A: Augereau \& Paploizou 2004).

Although low-mass M-dwarfs are the most populous (70\%) stars in the Galaxy, they have so far received little attention, mostly because their low luminosity makes the thermal emission and scattered light from their disks more difficult to detect. In a

\footnotetext{
1 http://astro. berkeley.edu/ kalas/disksite/pages/ gallery.html
} 
SCUBA survey of young stars of the $\beta$ Pic moving group and of the Local Association, Liu et al. (2004) detected the first two debris disks around M-dwarfs, AU Mic, and GJ182. Using SCUBA and MAMBO-2, Lestrade et al. (2006) surveyed 32 relatively young M-dwarfs of moving groups and newly detected one disk around the M 0.5 dwarf GJ842.2. Using Spitzer, Gautier et al. (2007) surveyed 62 nearby M-dwarfs at $24 \mu \mathrm{m}$, and subsamples of 41 at $70 \mu \mathrm{m}$ and of 20 at $160 \mu \mathrm{m}$, and found no firm detection. AU Mic was also imaged in scattered light as an edge-on and structured disk (Liu 2004; Krist et al. 2005).

Here we present new MAMBO-2 observations of nearby Mdwarfs, and combined them with our previous survey to analyze a total sample of $50 \mathrm{M}$-dwarfs (Table 1) in terms of their cold debris disk abundance.

We present the new M-dwarfs surveyed in Sect. 2, we describe the observations in Sect. 3, and results for both debris disks and background sources in the fields of some of the Mdwarfs in Sect. 4. Finally, we compare the detected fraction of cold debris disks around the M-dwarfs of our sample with the fractions for other stellar types to discuss whether or not the characteristics of M-dwarfs - mass and luminosity lower than solar - impact the formation of debris disks around them or their detectability.

\section{Sample of newly observed M-type dwarfs}

To complement our first survey of M-dwarfs whithin moving groups of ages $<600 \mathrm{Myr}$ (Lestrade et al. 2006), we observed the most nearby M-dwarfs, irrespective of age. We selected single M-dwarfs at a distance of less than $6 \mathrm{pc}$ and $\delta>-11^{\circ}$, and added five M-dwarfs binaries and six single M-dwarfs between 6 and $10 \mathrm{pc}$ that are in common with the Spitzer survey by Gautier et al. (2007). The five binaries in our sample are: GJ725 (M 3 and M 3.5) separated by 15" (53 AU), GJ234 (M 4.5 and M 8) separated by $1^{\prime \prime}$ (5 AU), GJ412 (M 2 and M 6) separated by 32" (160 AU), GJ569 (M 2.5 and M 8.5) separated by $1^{\prime \prime}$ (10 AU), and GJ65 (M 5.5 and M 5.5) separated by $2^{\prime \prime}$ (5.2 AU). These angular separations are so small that a single MAMBO-2 map can cover both components. The ages of the targeted near-by M-dwarfs are presently unknown.

\section{Observations}

The diameter usually adopted for debris disks is $120 \mathrm{AU}$, which for near-by stars is larger than the IRAM 30-m telescope beam of $10.7^{\prime \prime} F W H M$ at $\lambda=1.2 \mathrm{~mm}$. We therefore imaged each field with the 117-channel Max-Planck Bolometer array (MAMBO2; Kreysa et al. 1998) of the $30 \mathrm{~m}$ telescope on Pico Veleta, Spain $(2900 \mathrm{~m})$. MAMBO-2 has a half-power spectral bandwidth from 210 to $290 \mathrm{GHz}$, with an effective frequency centered on $250 \mathrm{GHz}(1.20 \mathrm{~mm})$ for thermal emission spectra. The effective FWHM beam is $10.7^{\prime \prime}$ and the undersampled field of view of the array is $4^{\prime}$. We used the standard on-the-fly mapping technique, where one map consists of 41 azimuthal subscans of $60 \mathrm{~s}$ each, with a scanning velocity of $4^{\prime \prime} / \mathrm{s}$ and an elevation incremental step of $4^{\prime \prime}$, while chopping the secondary mirror at $2 \mathrm{~Hz}$ by $60^{\prime \prime}$ in azimuth. The bolometers are arranged in a hexagonal pattern with a beam separation of $22^{\prime \prime}$. This scanning pattern produces time streams of data that are converted into a fully sampled spatial map with 3.5" pixels. Our observations were completed within pooled observing runs spread over the winter and summer periods from 2005 to 2007 . Atmospheric conditions were generally good during the observations, with typical zenith opacities between 0.1 and 0.3 at $250 \mathrm{GHz}$ and low sky noise. The telescope pointing was checked before and after each map by using the same nearest bright point source, and was found to be stable to greater accuracy than $3^{\prime \prime}$, except on a few occasions for which we discarded the data. The absolute flux calibration is based on observations of several standard calibration sources, including planets, and on a tipping curve (sky dip) measurement of the atmospheric opacity once every few hours. The resulting absolute flux calibration uncertainty is estimated to be about $10 \%$ (rms).

The data were analyzed using the mopsic software package written by R. Zylka at IRAM. The double-beam maps were combined to a single map using the shift-and-add procedure. Compared to a proper image restoration, this produces maps with about a factor 2 higher sensitivity, at the expense of no sensitivity to emission structures in scan direction that are larger that the wobbler throw of $60^{\prime \prime}$. In our sample, the shortest integration time per field is $30 \mathrm{~min}$, yielding an rms noise level of $\sim 2.5 \mathrm{mJy} / 11^{\prime \prime}$ beam in the central part of the map $\left(r<60^{\prime \prime}\right)$, steadily rising to $\sim 5 \mathrm{mJy} / 11^{\prime \prime}$ beam at its edge $\left(r \sim 140^{\prime \prime}\right)$. This non-uniform noise across the maps exists because the scanned field is about twice as large as the bolometer array size, so that more data is taken in the central part of the map than near the edges. Because of this non-uniformity, it is more judicious to present Signal-to-Noise $(\mathrm{S} / \mathrm{N})$ maps rather than intensity maps. The longest observation, by far, had a $20 \mathrm{~h} \mathrm{du}-$ ration and was targeted at GJ628, yielding an rms noise level of $\sim 0.5 \mathrm{mJy} / 11^{\prime \prime}$ beam in the central part of the map and $\sim 1.2 \mathrm{mJy} / 11^{\prime \prime}$ beam at $r \sim 140^{\prime \prime}$. The sky area covered by each map corresponds to $\sim 1700$ independent antenna beams. The residual noise in the map was found to be nearly Gaussian. Hence, a $4 \sigma$ detection in our maps is statistically significant. Below, we describe the procedure we used to extract discrete sources and to search for extended emission.

\section{Results}

Although initially intended, our complete survey of 50 M-dwarfs turns out not to be flux-limited, which makes its statistical interpretation not straightforward. First, we present the discrete millimeter sources detected in four MAMBO-2 maps (Figs. 1-3, and Table 2), and we discuss the nature of the intriguing cluster of sources around GJ526. Second, we present the deep search for faint debris disks made by averaging intensities over an effective area in the 42 MAMBO-2 maps of our complete survey (Tables 3 and 4). The 8 other M-dwarfs were observed in wide photometry mode with SCUBA (Table 5) and were already discussed by Lestrade et al. (2006). We use the complete sample of $50 \mathrm{M}$-dwarfs to estimate the fraction of cold debris disks around M-dwarfs and upper limits to their fractional dust luminosities. Three stars (GJ285, GJ393 and GJ4247) are in common between Tables 3-5.

\subsection{Discrete sources and structure around GJ526}

\subsubsection{Source extraction}

In Table 2, we summarize the characteristics of the discrete sources detected with Signal-to-Noise $S / N>4$ in the 42 fields mapped with MAMBO-2, each being $\sim 400^{\prime \prime} \times 400^{\prime \prime}$ in size and being centered on the position of an M-dwarf. The source extraction was done by searching each map for pixels with $S / N>4$ to select a block of $7 \times 7$ pixels centered on each of them. We then minimized $\chi^{2}$ between the 2-D Gaussian $F \times \exp \left(-0.5 \times\left[\left(x-x_{0}\right)^{2}+\left(y-y_{0}\right)^{2}\right] / \sigma^{2}\right)$ and the measured 
Table 1. The 50 M-dwarfs of our (sub)mm surveys.

\begin{tabular}{|c|c|c|c|c|c|c|}
\hline Name & Spectral & Binarity & $\begin{array}{l}\text { Selection } \\
\text { criterium }\end{array}$ & $\begin{array}{c}\text { Observed in } \\
\text { survey \# }\end{array}$ & $\begin{array}{l}\text { Bolometer } \\
\text { arrays }\end{array}$ & Publication \\
\hline GJ82 & M4 & Single & $20-150 \mathrm{Myr}$ & I & SCUBA & Lestrade et al. (2006) \\
\hline GJ212 & M0.5 & $"$ & $"$ & I & $"$ & $"$ \\
\hline GJ507.1 & M1.5 & $"$ & $"$ & I & " & $"$ \\
\hline GJ696 & M0 & $"$ & $"$ & I & ” & $"$ \\
\hline GJ876 & M4 & $"$ & $"$ & I & MAMBO & $"$ \\
\hline GJ628 & M3.5 & $"$ & $"$ & I & " & $"$ \\
\hline GJ402 & M4 & $"$ & $"$ & $\mathrm{I}$ & ” & $"$ \\
\hline GJ234 & $\mathrm{M} 4.5+\mathrm{M} 8$ & Binary & $"$ & I \& II & $"$ & Lestrade et al. (2006) and this work \\
\hline GJ285 & M4.5 & Single & $"$ & I & SCUBA \& MAMBO & Lestrade et al. (2006) \\
\hline GJ393 & M2 & $"$ & $"$ & I & $"$ & $"$ \\
\hline GJ9809 & M0 & $"$ & " & I & $"$ & $"$ \\
\hline GJ875.1 & M3 & $"$ & $35-55 \mathrm{Myr}$ & I & SCUBA & $"$ \\
\hline GJ856A & M3 & $"$ & $100-125 \mathrm{Myr}$ & I & MAMBO & $"$ \\
\hline GJ277B & M3.5 & $"$ & $200 \mathrm{Myr}$ & I & SCUBA & $"$ \\
\hline GJ842.2 & M0.5 & $"$ & $"$ & I & " & $"$ \\
\hline GJ890 & M2 & $"$ & $"$ & I & ” & $"$ \\
\hline GJ1111 & M6.5 & $"$ & $"$ & I \& II & MAMBO & Lestrade et al. (2006) and this work \\
\hline GJ408 & M2.5 & $"$ & $"$ & I \& II & $"$ & $"$ \\
\hline GJ4247 & M4 & $"$ & $"$ & I \& II & SCUBA \& MAMBO & $"$ \\
\hline GJ447 & M4.0 & $"$ & $500 \mathrm{Myr}$ & I \& II & MAMBO & $"$ \\
\hline GJ625 & M1.5 & $"$ & $"$ & I \& II & " & $"$ \\
\hline GJ569 & $\mathrm{M} 2.5+\mathrm{M} 8.5$ & Binary & $"$ & I \& II & 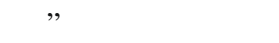 & $"$ \\
\hline GJ873 & M3.5 & Single & $"$ & I & $"$ & Lestrade et al. (2006) \\
\hline GJ65 & M5.5+M5.5 & Binary & $600 \mathrm{Myr}$ & I & $"$ & $"$ \\
\hline GJ3379 & M4 & Single & , & I & $"$ & $"$ \\
\hline GJ849 & M3.5 & " & $"$ & I & $"$ & " \\
\hline GJ791.2 & M4.5 & $"$ & $"$ & I & $"$ & $"$ \\
\hline GJ109 & M3.5 & 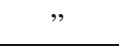 & $"$ & I \& II & " & Lestrade et al. (2006) and this work \\
\hline GJ699 & M4.0 & Single & $1.82 \mathrm{pc}$ & II & MAMBO & this work \\
\hline GJ406 & M6.0 & ", & $2.38 \mathrm{pc}$ & II & " & $"$ \\
\hline GJ411 & M2.0 & $"$ & $2.54 \mathrm{pc}$ & II & $"$ & $"$ \\
\hline GJ905 & M5.5 & $"$ & $3.16 \mathrm{pc}$ & II & $"$ & $"$ \\
\hline GJ725 & M3+M3.5 & Binary & $3.57 \mathrm{pc}$ & II & $"$ & $"$ \\
\hline GJ54.1 & M4.5 & Single & $3.72 \mathrm{pc}$ & II & $"$ & $"$ \\
\hline GJ273 & M3.5 & $"$ & $3.79 \mathrm{pc}$ & II & $"$ & $"$ \\
\hline GJ83.1 & M4.5 & $"$ & $4.44 \mathrm{pc}$ & II & $"$ & $"$ \\
\hline GJ687 & M3.0 & $"$ & $4.53 \mathrm{pc}$ & II & $"$ & $"$ \\
\hline LHS292 & M6.5 & $"$ & $4.54 \mathrm{pc}$ & II & $"$ & $"$ \\
\hline GJ1002 & M5.5 & $"$ & $4.69 \mathrm{pc}$ & II & $"$ & $"$ \\
\hline GJ412 & $\mathrm{M} 2+\mathrm{M} 6$ & Binary & $4.83 \mathrm{pc}$ & II & $"$ & $"$ \\
\hline GJ388 & M3.0 & Single & $4.89 \mathrm{pc}$ & II & $"$ & $"$ \\
\hline GJ445 & M3.5 & $"$ & $5.38 \mathrm{pc}$ & II & $"$ & $"$ \\
\hline LHS1723 & M4.5 & $"$ & $5.43 \mathrm{pc}$ & II & " & $"$ \\
\hline GJ526 & M1.5 & $"$ & $5.43 \mathrm{pc}$ & II & $"$ & $"$ \\
\hline GJ251 & M3.0 & $"$ & $5.57 \mathrm{pc}$ & II & $"$ & $"$ \\
\hline GJ205 & M1.5 & $"$ & $5.71 \mathrm{pc}$ & II & $"$ & $"$ \\
\hline GJ213 & M4.0 & $"$ & $5.87 \mathrm{pc}$ & II & $"$ & $"$ \\
\hline GJ908 & M1.0 & $"$ & $5.93 \mathrm{pc}$ & II & $"$ & $"$ \\
\hline GJ581 & M3 & $"$ & $6.27 \mathrm{pc}$ & II & $"$ & $"$ \\
\hline GJ102 & M4 & $"$ & $7.75 \mathrm{pc}$ & II & $"$ & $"$ \\
\hline
\end{tabular}

Survey I was presented in Lestrade et al. (2006) and its data are included in the statistical analysis of the present paper. Survey II is based on new data with MAMBO-2 presented in the present paper. Selection criteria are ages $<600$ Myr for survey I and the nearest M-dwarfs but of undetermined ages for survey II. Ages are based on Moving Groups (Local Association (20-150 Myr), IC 2391 (35-55 Myr), AB Dor (100-125 Myr), Castor (200 Myr), Ursa Maj (500 Myr) and Hyades (600 Myr)) identified by Montes et al. (2001) and Zuckerman \& Inseok Song (2004a,b). Some stars were observed in both surveys.

intensities over this block by varying the peak flux $F$, the parameter $\sigma(F W H M=2.35 \sigma)$, and the peak position $\left(x_{0}, y_{0}\right)$ by less than a pixel from the $S / N>4$ pixel. We used the mean brightness computed from the 32 pixels surrounding the $7 \times 7$ block to remove any constant level. With this scheme, this level is determined from pixels that are far enough from the 2-D Gaussian peak to be at the map floor, since the Gaussian $F W H M$ is 3 to
5 pixels $\left(11^{\prime \prime}-17^{\prime \prime}\right)$ for our sources. This correction amounted to between $5 \%$ and $20 \%$ of the integrated flux density. The integrated flux densities of sources in Table 2 are $S_{\text {int }}=2 \pi \sigma F$. We extracted 13 discrete sources with $S / N>4$ for $F$ in 4 fields out of the 42 MAMBO-2 maps; 8 sources were newly found in the fields of GJ526, GJ725, and GJ569. They are all shown in Figs. 1-3. The 5 other sources are in the field of GJ628 as already 
Table 2. Millimeter sources found in our MAMBO-2 maps.

\begin{tabular}{|c|c|c|c|c|c|c|}
\hline Source name & Star Field & 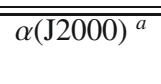 & 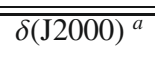 & $\begin{array}{c}\text { Integrated flux density }^{b} \\
\text { at } 1.2 \mathrm{~mm}(\mathrm{mJy})\end{array}$ & $S / N$ & $\begin{array}{l}\text { Source } \\
F W H M\end{array}$ \\
\hline MM145428+160439 & GJ569 & 145428.2 & 160439 & $5.3 \pm 1.1$ & 4.9 & $11^{\prime \prime}$ \\
\hline MM184222+593828 & GJ725 & $\begin{array}{lll}18 & 42 & 22.7\end{array}$ & $\begin{array}{lll}59 & 38 & 28\end{array}$ & $30-63^{c}$ & $6.7-7.3$ & $13^{\prime \prime}-15^{\prime \prime}$ \\
\hline MM184253+593756 & GJ725 & $\begin{array}{lll}18 & 42 & 53.3\end{array}$ & $5937 \quad 56$ & $9.7 \pm 1.9$ & 5.1 & $11^{\prime \prime}$ \\
\hline MM134539+145139 & GJ526 & $\begin{array}{lll}13 & 45 & 39.0\end{array}$ & $1451 \quad 39$ & $3.2 \pm 0.7$ & 4.4 & $11^{\prime \prime}$ \\
\hline MM134540+145446 & GJ526 & $\begin{array}{lll}13 & 45 & 40.7\end{array}$ & $1454 \quad 46$ & $4.3 \pm 0.8$ & 5.2 & $11^{\prime \prime}$ \\
\hline MM134541+145417 & GJ526 & $13 \quad 45 \quad 41.3$ & $1454 \quad 17$ & $6.3 \pm 1.0$ & 6.1 & $11^{\prime \prime}$ \\
\hline MM134543+145317 & GJ526 & $\begin{array}{lll}13 & 45 & 43.1\end{array}$ & $\begin{array}{lll}14 & 53 \quad 17\end{array}$ & $3.0 \pm 0.7$ & 4.9 & $11^{\prime \prime}$ \\
\hline MM134546+145240 & GJ526 & $13 \quad 45 \quad 46.7$ & 145240 & $5.6 \pm 0.7$ & 8.6 & $11^{\prime \prime}$ \\
\hline MM163007-123942 & GJ628 ${ }^{d}$ & $1630 \quad 07.6$ & $\begin{array}{lll}-12 & 39 & 42\end{array}$ & $7.3 \pm 1.2$ & 6.2 & $14 \pm 4^{\prime \prime}$ \\
\hline MM163022-123925 & GJ628 ${ }^{e}$ & 163022.3 & $\begin{array}{lll}-12 & 39 & 25\end{array}$ & $3.8 \pm 0.9$ & 4.4 & $17 \pm 5^{\prime \prime}$ \\
\hline MM163019-123830 & GJ628 ${ }^{f}$ & $\begin{array}{lll}16 & 30 & 19.7\end{array}$ & $\begin{array}{lll}-12 & 38 & 30\end{array}$ & $4.1 \pm 0.8$ & 4.9 & $11^{\prime \prime}$ \\
\hline MM163015-123911 & $\mathrm{GJ}_{628^{g}}$ & $1630 \quad 15.6$ & $\begin{array}{lll}-12 & 39 & 11\end{array}$ & $4.8 \pm 0.8$ & 6.2 & $11^{\prime \prime}$ \\
\hline MM163013-124057 & GJ628 ${ }^{h}$ & $\begin{array}{lll}16 & 30 & 13.7\end{array}$ & $\begin{array}{lll}-12 & 40 & 57\end{array}$ & $4.3 \pm 1.0$ & 4.8 & $11^{\prime \prime}$ \\
\hline
\end{tabular}

${ }^{a}$ The MAMBO coordinate uncertainties are $\sim 3.5^{\prime \prime} .{ }^{b}$ Flux density (mJy) is integrated under the fitted 2D-Gaussian. ${ }^{c}$ Flux density is provided as lower and upper limits because the source is located close to one of the borders of the map where some systematics remain in the data (see text). ${ }^{d},{ }^{e},{ }^{f},{ }^{g},{ }^{h}$ These sources were found in survey I; ${ }^{d}$ is GJ628-W, ${ }^{e}$ is GJ628-E, ${ }^{f}$ is GJ628-NE, ${ }^{g}$ is GJ628-NW, and ${ }^{h}$ is GJ628-SW in Fig. 2 and Table 3 of Lestrade et al. (2006). Note that there are typographic errors in the minute column of declinations in Table 3 of Lestrade et al. (2006). The positions of the GJ628 sources given now are correct and supersede this first publication. Also, the flux densities in this first publication were peak flux densities while they are integrated flux densities now.

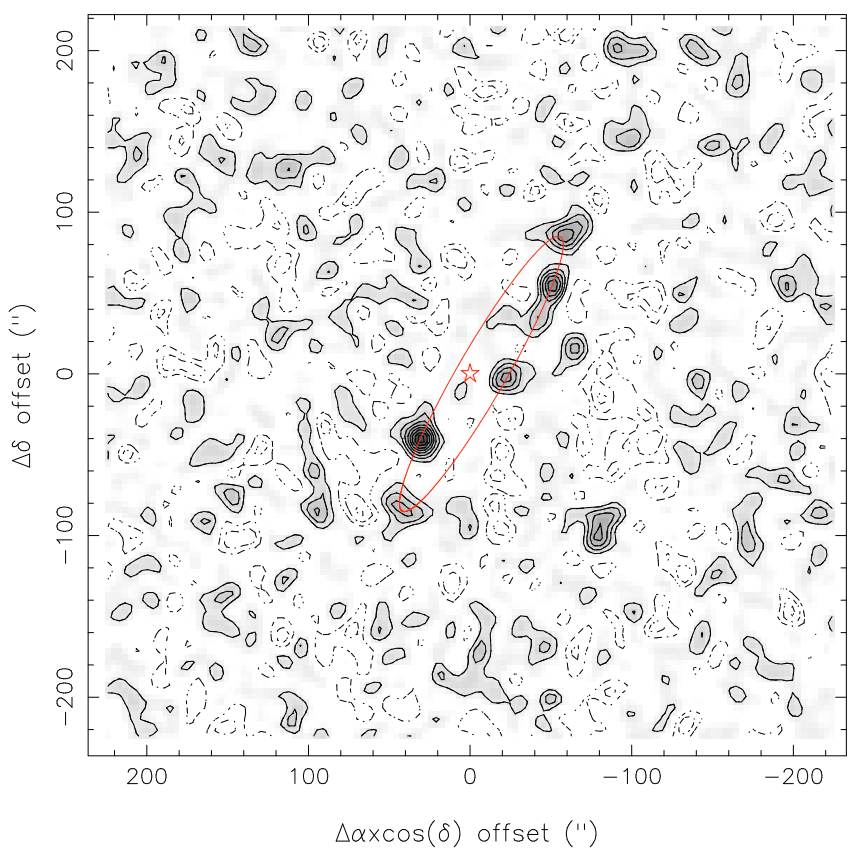

Fig. 1. MAMBO-2 Signal-to-noise ratio map of the field around the M1.5 dwarf GJ526 at $\lambda=1.2 \mathrm{~mm}$. The pixel size is $3.5^{\prime \prime} \times 3.5^{\prime \prime}$. The noise $\mathrm{rms}$ is $\sigma=0.58 \mathrm{mJy} / 11^{\prime \prime}$ beam in the central region $\left(r<60^{\prime \prime}\right)$ and increases towards the edges of the map, $\sim 1.3 \mathrm{mJy} / 11^{\prime \prime}$ beam at $r \sim 140^{\prime \prime}$. The contours are $-4 \sigma,-3 \sigma,-2 \sigma,-1 \sigma$ (dotted lines), and $1 \sigma, 2 \sigma, 3 \sigma, 4 \sigma, 5 \sigma, 6 \sigma, 7 \sigma, 8 \sigma$. The ellipse $\left(a=98^{\prime \prime}, b=15^{\prime \prime}\right.$, and $\mathrm{PA}=-30^{\circ}$ ) is almost centered on the star position and connects five sources that might be clumps of a debris disk inclined to the plane of the sky by $81^{\circ}$ (see Sect. 4.1.3). The field is centered on the position of GJ526 of early 2006: $\alpha(\mathrm{J} 2000)=13^{\mathrm{h}} 45^{\mathrm{m}} 44.52^{\mathrm{s}}$ and $\delta(\mathrm{J} 2000)=$ $14^{\circ} 53^{\prime} 20.6^{\prime \prime}$ (red star). See Table 2 for integrated flux densities and coordinates of sources.

reported in Lestrade et al. (2006); typographic errors in their coordinates in this first publication are corrected in Table 2. In this

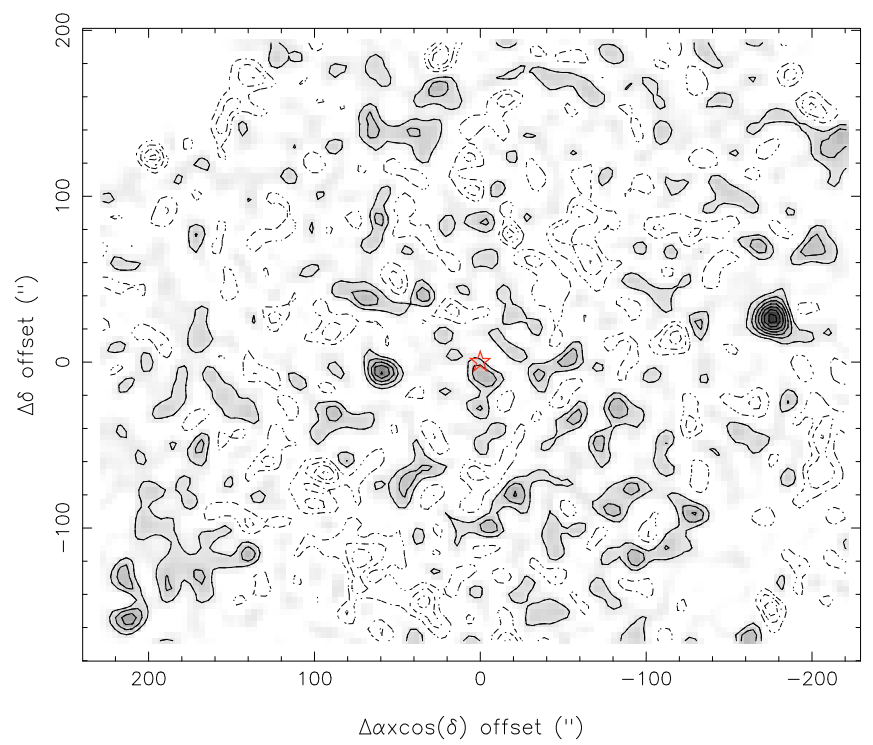

Fig. 2. MAMBO-2 Signal-to-noise ratio map of the field around the binary GJ725 (M3 + M3.5 dwarfs) at $\lambda=1.2 \mathrm{~mm}$. The pixel size is $3.5^{\prime \prime} \times$ $3.5^{\prime \prime}$. The noise rms is $\sigma=1.4 \mathrm{mJy} / 11^{\prime \prime}$ beam in the central region $(r<$ $\left.60^{\prime \prime}\right)$ and increases towards the edges of the map, $\sim 4.3 \mathrm{mJy} / 11^{\prime \prime}$ beam at $r \sim 170^{\prime \prime}$. The contours are $-4 \sigma,-3 \sigma,-2 \sigma,-1 \sigma$ (dotted lines), and $1 \sigma, 2 \sigma, 3 \sigma, 4 \sigma, 5 \sigma, 6 \sigma, 7 \sigma$. The source MM184222+593828 westward has an integrated flux density between 30 and $63 \mathrm{mJy}(S / N \sim 7)$. The other source, MM184253+593756, has an integrated flux density of $9.7 \mathrm{mJy}$ and a $S / N$ of 5.1. The field is centered on the position of GJ725A of early 2006: $\alpha(\mathrm{J} 2000)=18^{\mathrm{h}} 42^{\mathrm{m}} 45.48^{\mathrm{s}}$ and $\delta(\mathrm{J} 2000)=$ $59^{\circ} 38^{\prime} 01.7^{\prime \prime}$ (red star). See Table 2 for integrated flux densities and coordinates of the sources.

table, the lowest integrated flux density is $3.0 \pm 0.7 \mathrm{mJy}$ and the highest is between 30 and $63 \mathrm{mJy}$. There is no optical counterpart within 7" ( $\sigma \sigma$ MAMBO-2 position error) of any of these sources in the USNO-B1 catalogue (Monet et al. 2003), except for MM163007-123942 (GJ628W) at the $1 \sigma$ level in position 


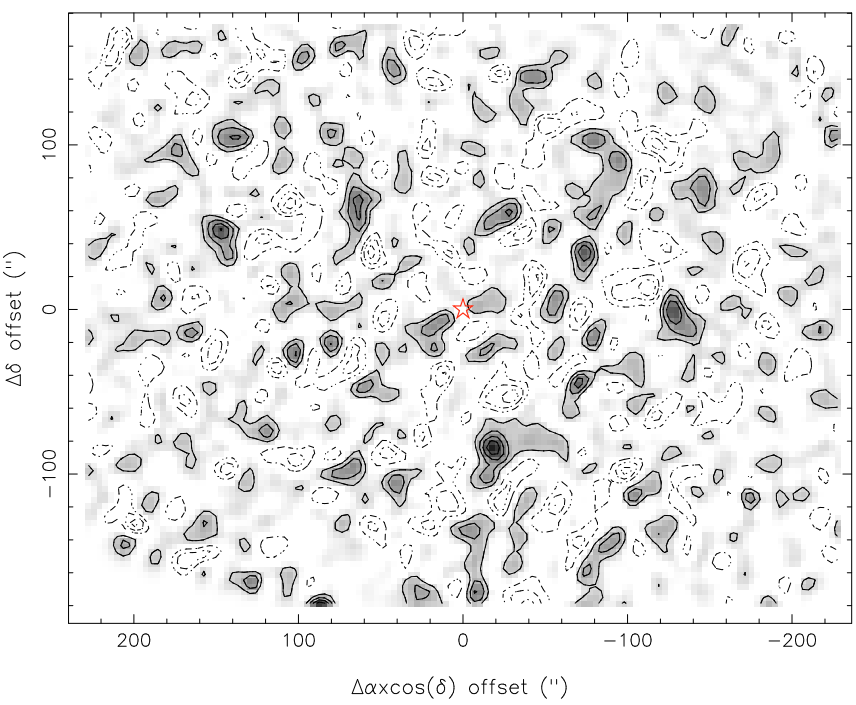

Fig. 3. MAMBO-2 Signal-to-noise ratio map of the field around the binary GJ569 (M $2.5+$ M 8.5 dwarfs) at $\lambda=1.2 \mathrm{~mm}$. The pixel size is $3.5^{\prime \prime} \times 3.5^{\prime \prime}$. The noise rms is $\sigma=1.1 \mathrm{mJy} / 11^{\prime \prime}$ beam in the central region $\left(r<60^{\prime \prime}\right)$ and increases towards the edges of the map, $\sim 2.8 \mathrm{mJy} / 11^{\prime \prime}$ beam at $r \sim 140^{\prime \prime}$. The contours are $-4 \sigma,-3 \sigma,-2 \sigma,-1 \sigma$ (dotted lines), and $1 \sigma, 2 \sigma, 3 \sigma, 4 \sigma$. The source MM145428+160439 has an integrated flux density of $5.3 \mathrm{mJy}$ and a $S / N$ of 4.9 . The field is centered on the position of GJ569A of early 2006: $\alpha(\mathrm{J} 2000)=$ $14^{\mathrm{h}} 54^{\mathrm{m}} 29.35^{\mathrm{s}}$ and $\delta(\mathrm{J} 2000)=16^{\circ} 06^{\prime} 03.2^{\prime \prime}$ (red star). See Table 2 for integrated flux density and coordinates of the source.

(object I mag $=18.92$ ) as already reported in Lestrade et al. (2006). Additionally, we searched the fields of GJ526 and GJ569 that were imaged in the Sloan Digital Sky Survey (SDSS). In the field of GJ526, there is only one SDSS object $(z$ mag $=20.4)$ which is as close as $7^{\prime \prime}$ from MM134540+145446. However, the confusion limit is reached over such a separation since the density of SDSS objets is $\sim 22000$ objects $/ \mathrm{deg}^{2}$ as measured over the $4^{\prime} \times 4^{\prime}$ area centered on the star position. In the field of GJ569 ( 33000 SDSS objects/deg $\left.{ }^{2}\right)$, there are two SDSS objets (mag $u=19.9, g=21.6$ ) as close as $8^{\prime \prime}$ from MM145428+160439, but again confusion prevents identification with the MAMBO-2 $\mathrm{m}$ source. We found no radio counterpart within the $3 \sigma$ MAMBO-2 position uncertainty $\left(10.5^{\prime \prime}\right)$ in the NVSS catalogue (Condon et al. 1998) to the flux density limit of $2.5 \mathrm{mJy}$ at $1.4 \mathrm{GHz}$, except for MM163007-123942 $(\mathrm{GJ} 628 \mathrm{~W})$ at the $1.7 \sigma$ level in position (object flux density = $5.6 \pm 0.5 \mathrm{mJy}$ ) as already reported in Lestrade et al. (2006). Finally, we found no IR counterpart within the $3 \sigma$ MAMBO2 position uncertainty in the 2MASS All-Sky Catalog of Point Sources (Skrutskie et al. 2006).

\subsubsection{A bright millimeter source}

The source MM184222+593828 in the field of GJ725 has a flux density of between 30 and $63 \mathrm{mJy}$ (Table 2), which is bright compared to millimeter sources found previously in empty fields mapped with MAMBO-2 (Greve et al. 2004; Bertoldi et al. 2007; and Voss et al. 2006). These lower and upper limits were obtained by using different atmospheric correlation lengths in the skynoise reduction within the mopsic data reduction package, yielding similar detections of $S / N \sim 7$. Systematics affects the accuracy of the flux measurement for this source, which is near the edge of the map. This source is possibly resolved with a source $F W H M$ between $12^{\prime \prime}$ and $15^{\prime \prime}$ depending on the reduction parameter used. Although this is rare, it has been shown that submm galaxies can have multiple components (Tacconi et al. 2008). We shall discuss this source together with complementary observations in a forthcoming paper.

\subsubsection{Structure around GJ526}

The map of GJ526 is shown in Fig. 1. There are five sources that can be connected by an ellipse almost centered on the star; precisely four robust ones with $S / N>4$ and an additional one, farthest to the SE, that is only detected with $S / N>3.5$. This source is possibly real since statistically less than 1 positive noise peak is expected at this level over the whole map if dominated by Gaussian noise. To estimate whether this is the case or not, we identified the negative noise spikes $S / N<-3$ and the positive spikes $3<S / N<4$ in our $440^{\prime \prime} \times 420^{\prime \prime}$ map ( 2050 beams). The total of these 7 noise spikes found in the map is consistent with the 6 theoretically expected for $|S / N|>3$ for Gaussian noise. Also, the detection of the SE source is robust since it appears at the same position in the two independent maps we made by dividing the data in half. There is also a $\sim 3 \sigma$ source to the West close to the ellipse but its detection is not robust to data selection. Finally, there is an $S / N>4$ source farther away in the SW direction.

The ellipse is offset by two pixels in right ascension from the position of the star, its semi-major and semi-minor axes are $a=98^{\prime \prime}$ and $b=15^{\prime \prime}$, and its orientation is $\mathrm{PA}=-30^{\circ}$. This structure might be the projected ring of a clumpy debris disk, its inclination being $81^{\circ}$ from the plane of the sky. The offset between the star and the center of the ellipse is only 2 pixels and might be caused by position uncertainties or/and by true source structure. However, the 5 connected sources do not appear to be embedded in any extended emission as expected for a debris disk. The mean brightness over a rectangular box $220^{\prime \prime} \times 40^{\prime \prime}$ (92 beams), oriented at $\mathrm{PA}=-30^{\circ}$, and centered on the star is $0.21 \pm 0.073 \mathrm{mJy} / 11^{\prime \prime}$ beam, i.e., $\sim 3 \sigma$. This mean brightness matches the mean of the 5 flux densities of the connected sources within the box, indicating that any extended emission must have a brightness $<3 \times 0.073 \mathrm{mJy} / 11^{\prime \prime}$ beam at $1.2 \mathrm{~mm}$.

We now test whether or not such a source cluster can be produced by the distribution of background sources. We carry out a first test to estimate the probability that the connected sources around GJ526 can cluster as tightly as they do in Fig. 1 if they were background sources. For this test, we use the statistical analysis of spatial point patterns developed by Diggle (2003). This analysis is based on the nearest neighbour distance, defined as the distance between a point (a source for us) and its nearest neighbour. For GJ526, all the nearest neighbour distances for the 5 sources connected by the ellipse are $<60^{\prime \prime}$. The probability that $k$ distances $x$ be $<60^{\prime \prime}$ among $N$ distances if the mean frequence of occurrence for $x<60^{\prime \prime}$ over the whole sky is $f$, is given by the binomial distribution $B_{k}=\left(\begin{array}{l}N \\ k\end{array}\right) f^{k}(1-f)^{N-k}$. We can derive the mean frequence of occurrence $f$ from the three empty fields (ELAIS N2, Lockman Hole, and COSMOS) maped by MAMBO-2 (Greves et al. 2004; and Bertoldi et al. 2007). We found that there are 9 distances $<60^{\prime \prime}$ between the 71 sources of these three fields, and so $f=9 /(71-3)=0.13$. The factor $(71-3)$ is the number of nearest neighbour distances for 71 sources distributed in the 3 separate fields. For the five sources around GJ526 in Fig. 1, all 5-1 nearest neighbour distances are $<60^{\prime \prime}$ as already mentioned, so $k=N=4$ and the probability $B_{k}$ that background sources can produce such a number of small distances is as low as $0.1 \%$. 
Table 3. Search for debris disks around nearby M-dwarfs with our new MAMBO-2 data.

\begin{tabular}{|c|c|c|c|c|c|c|c|c|}
\hline Name & $\begin{array}{l}\text { Spectral } \\
\text { type }\end{array}$ & $\begin{array}{c}D \\
(\mathrm{pc})\end{array}$ & $\begin{array}{c}\text { Gal. } 1 . \\
\left(^{\circ}\right)\end{array}$ & $\begin{array}{l}\text { Integration } \\
\text { time } \\
\text { (h) }\end{array}$ & $\begin{array}{c}\text { Map rms } \\
\text { (mJy/11" beam) } \\
\text { (1) }\end{array}$ & $\begin{array}{c}\text { Mean brightness } \\
\mu \pm \sigma_{\mu} \\
\text { (mJy/1 } 11^{\prime \prime} \text { beam) } \\
\text { (2) }\end{array}$ & $\begin{array}{c}\mu / \sigma_{\mu} \\
(2)\end{array}$ & $\begin{array}{c}\sigma \sigma \text { flux density } \\
\text { upper limit }(1.2 \mathrm{~mm}) \\
(\mathrm{mJy}) \\
(3)\end{array}$ \\
\hline GJ699 & M4.0 & 1.82 & 14 & 1.5 & 1.22 & $-0.03 \pm 0.08$ & -0.36 & $<7.9$ \\
\hline GJ406 & M6.0 & 2.38 & 56 & 2.5 & 0.99 & $-0.18 \pm 0.08$ & -2.29 & $<4.9$ \\
\hline GJ411 & M2.0 & 2.54 & 65 & 6.5 & 0.67 & $0.06 \pm 0.06$ & 0.97 & $<3.1$ \\
\hline GJ905 & M5.5 & 3.16 & -17 & 0.5 & 2.30 & $0.09 \pm 0.24$ & 0.38 & $<8.6$ \\
\hline GJ447 & M4.0 & 3.34 & 59 & 1.5 & 1.19 & $-0.09 \pm 0.13$ & -0.69 & $<4.2$ \\
\hline GJ725(*) & $\mathrm{M} 3+\mathrm{M} 3.5$ & 3.57 & 24 & 1.0 & 1.44 & $0.29 \pm 0.17$ & 1.69 & $<4.7$ \\
\hline GJ1111 & M6.5 & 3.62 & 32 & 1.0 & 1.75 & $0.09 \pm 0.20$ & 0.44 & $<5.7$ \\
\hline GJ54.1 & M4.5 & 3.72 & -79 & 1.5 & 1.49 & $-0.33 \pm 0.18$ & -1.86 & $<4.7$ \\
\hline GJ273 & M3.5 & 3.79 & 10 & 0.5 & 2.13 & $0.17 \pm 0.25$ & 0.68 & $<6.6$ \\
\hline GJ234 & M4.5+M8 & 4.12 & -6 & 2.5 & 1.03 & $0.30 \pm 0.13$ & 2.23 & $<2.9$ \\
\hline GJ83.1 & M4.5 & 4.44 & -46 & 1.5 & 1.40 & $-0.37 \pm 0.18$ & -2.11 & $<3.7$ \\
\hline GJ687 & M3.0 & 4.53 & 32 & 4.0 & 0.76 & $0.25 \pm 0.11$ & 2.18 & $<2.9$ \\
\hline LHS292 & M6.5 & 4.54 & 41 & 0.5 & 2.29 & $-0.35 \pm 1.52$ & -0.23 & $<5.9$ \\
\hline GJ1002 & M5.5 & 4.69 & -69 & 0.5 & 2.23 & $0.14 \pm 0.33$ & 0.43 & $<5.6$ \\
\hline GJ412 & M2+M6 & 4.83 & 63 & 0.5 & 2.26 & $-0.21 \pm 0.36$ & -0.58 & $<5.4$ \\
\hline GJ388 & M3.0 & 4.89 & 54 & 5.0 & 0.73 & $0.20 \pm 0.06$ & 3.25 & $<1.8$ \\
\hline GJ445 & M3.5 & 5.38 & 38 & 1.0 & 1.55 & $0.05 \pm 0.28$ & 0.18 & $<3.4$ \\
\hline LHS1723 & M4.5 & 5.43 & -27 & 0.5 & 2.23 & $0.49 \pm 0.42$ & 1.17 & $<4.8$ \\
\hline GJ526(**) & M1.5 & 5.43 & 72 & 16.0 & 0.58 & $-0.02 \pm 0.12$ & -0.16 & $<1.4$ \\
\hline GJ251 & M3.0 & 5.57 & 15 & 1.0 & 1.54 & $0.14 \pm 0.29$ & 0.48 & $<1.7$ \\
\hline GJ205 & M1.5 & 5.71 & -19 & 1.0 & 1.86 & $-1.09 \pm 0.37$ & -2.93 & $<2.0$ \\
\hline GJ213 & M4.0 & 5.87 & -9 & 0.5 & 2.19 & $-0.31 \pm 0.44$ & -0.70 & $<2.4$ \\
\hline GJ908 & M1.0 & 5.93 & -57 & 0.5 & 2.01 & $0.24 \pm 0.39$ & 0.61 & $<2.2$ \\
\hline GJ581 & M3 & 6.27 & 40 & 0.5 & 1.96 & $-0.75 \pm 0.43$ & -1.75 & $<2.1$ \\
\hline GJ625 & M1.5 & 6.58 & 43 & 5.0 & 0.69 & $0.38 \pm 0.15$ & 2.53 & $<0.7$ \\
\hline GJ408 & $\mathrm{M} 2.5$ & 6.62 & 64 & 2.0 & 1.13 & $0.42 \pm 0.14$ & 3.05 & $<1.2$ \\
\hline GJ109 & M3.5 & 7.55 & -31 & 3.0 & 0.98 & $0.32 \pm 0.22$ & 1.48 & $<1.1$ \\
\hline GJ102 & M4 & 7.75 & -32 & 0.5 & 2.20 & $-0.09 \pm 0.60$ & -0.15 & $<2.4$ \\
\hline GJ4247 & M4 & 8.96 & -21 & 3.5 & 0.74 & $0.63 \pm 0.25$ & 2.56 & $<0.8$ \\
\hline GJ569 (*) & $\mathrm{M} 2.5+\mathrm{M} 8.5$ & 9.81 & 59 & 2.0 & 1.09 & $-0.46 \pm 0.37$ & -1.25 & $<1.2$ \\
\hline
\end{tabular}

(1) rms estimated for $r<60^{\prime \prime}$ in the map; (2) mean brightness and uncertainty of mean computed by averaging intensities over an effective disk of radius $60 \mathrm{AU}$; (3) the $3 \sigma$ flux density upper limit is computed also over the same effective area (radius = $60 \mathrm{AU})$. $(*)$ : background sources in map (see Table 2); (**): possibly a large debris disk (see Fig. 1 and Sect. 4.1.3).

As a second test, we use the elliptical pattern connecting 6 submm sources recognisable in the North-West part of the COSMOS field maped by MAMBO-2 and displayed in Fig. 2 of Bertoldi et al. (2007). This field is $20 \times 20 \mathrm{arcmin}^{2}$ in size and the mean occurrence of ellipse of major-axis $200^{\prime \prime}$, as in the field of GJ526, is $1 / 20 \times 20 \mathrm{arcmin}^{-2}$. We compute the Poisson probability of finding a similar ellipse in a field that we take as small as $200 \times 200 \operatorname{arcsec}^{2}$ to account for the ellipse around GJ526 being in an area restricted to the central part of the map, i.e., centered on the position of GJ525. This probability is $2.7 \%$. This test is only indicative because the COSMOS field has been mapped at the level of $1 \mathrm{mJy} /$ beam rms, while our map is twice as deep for the field around GJ526.

In summary, the five sources in Fig. 1 that are symmetrically located around GJ526 are connected by an ellipse almost centered on the star. The two tests carried out above provide indications that this structure is statistically inconsistent with known spatial distributions of background submm galaxies. Hence, at this stage, we cannot rule out the hypothesis that the sources are associated with the star. In this case, the 5 connected sources could be indicative of azimuthal structures in an inclined debris disk around GJ526, whose extended emission is not seen because the map is not deep enough. Complementary observations at $850 \mu \mathrm{m}$, and at shorter wavelengths with Herschel, should attempt to detect the extended emission of the disk. Eventually, astrometry should detect the same proper motion for the 5 connected sources as for the star GJ526 (2.3"/yr in the SE direction) if indeed they are part of a debris disk. The mid-epoch of our MAMBO-2 data is early 2007, so that the 5 sources should have moved in concert with the star by a full IRAM-30 m beam by 2011, providing definitive proof of a disk.

Such a debris disk would have a radius as large as $~ 500 \mathrm{AU}$ at the distance of GJ526. We examine whether or not this is conceivable. First, we note that exceptionally large debris disks, $520 \mathrm{AU}$ and $600 \mathrm{AU}$ in extent, have been found around the $184 \mathrm{Myr}$ old A0-type dwarf $\gamma$ Oph at $70 \mu \mathrm{m}$ (Su et al. 2008), and around the main sequence F8V $q^{1}$ Eri at $870 \mu \mathrm{m}$ (Liseau et al. 2008). Second, protoplanetary disks where planetesimals and planets form extend to almost $1000 \mathrm{AU}$ as observed for example around the young close binary GG Tau (Dutrey et al. 1994). In the model proposed by Kenyon \& Bromley (2004a), icy-planets successively form in waves outward in the disk producing larger and larger dusty rings from collisional cascades. In their model, the planet formation timescale is $15-20 \times\left(\Sigma_{0} / \Sigma_{M M S N}\right)^{-1}(a / 30 \mathrm{AU})^{3} \mathrm{Myr}$ in a quiet disk (their Eq. (4)). Assuming for $\Sigma_{0}$ as much as 15 times the surface density of the minimum-mass solar nebula $\Sigma_{M M S N}$ as required for the formation of Jupiter in the solar system (Lissauer 1987), the timescale for planet formation to reach $500 \mathrm{AU}$ in the GJ526 system is $\sim 5$ Gyr. The stellar diameter of GJ526 measured by the 
Table 4. Reanalysis of the MAMBO-2 data of the M-dwarfs associated with moving groups of survey I.

\begin{tabular}{|c|c|c|c|c|c|c|c|c|}
\hline Name & $\begin{array}{l}\text { Spectral } \\
\text { type }\end{array}$ & $\begin{array}{c}D \\
(\mathrm{pc})\end{array}$ & $\begin{array}{c}\text { Gal. } 1 . \\
\left({ }^{\circ}\right)\end{array}$ & $\begin{array}{l}\text { Integration } \\
\text { time } \\
\text { (hours) }\end{array}$ & $\begin{array}{c}\text { Map rms } \\
\text { (mJy/11" beam) } \\
\text { (1) }\end{array}$ & $\begin{array}{c}\text { Mean brightness } \\
\mu \pm \sigma_{\mu} \\
\text { (mJy/11" beam) } \\
\text { (2) }\end{array}$ & $\begin{array}{c}\mu / \sigma_{\mu} \\
(2)\end{array}$ & $\begin{array}{c}3 \sigma \text { flux density } \\
\text { upper limit }(1.2 \mathrm{~mm}) \\
(\mathrm{mJy}) \\
(3)\end{array}$ \\
\hline GJ65 & M5.5 & 2.6 & -76 & 1.0 & 2.30 & $-0.15 \pm 0.17$ & -0.87 & $<10.4$ \\
\hline GJ628 & M3.5 & 4.5 & 24 & 20.0 & 0.51 & $-0.06 \pm 0.08$ & -0.74 & $<1.3$ \\
\hline GJ876 & M4 & 4.7 & -59 & 0.5 & 2.80 & $0.17 \pm 0.46$ & 0.37 & $<7.0$ \\
\hline GJ873 & M3.5 & 5.0 & -13 & 0.5 & 2.31 & $0.33 \pm 0.38$ & 0.86 & $<5.4$ \\
\hline GJ3379 & M4 & 5.4 & -10 & 0.5 & 2.57 & $0.05 \pm 0.56$ & 0.09 & $<5.6$ \\
\hline GJ285 & M4.5 & 5.9 & 13 & 1.0 & 1.74 & $-0.88 \pm 0.35$ & -2.52 & $<1.9$ \\
\hline GJ402 & M4 & 6.8 & 55 & 1.0 & 1.81 & $-0.86 \pm 0.39$ & -2.18 & $<2.0$ \\
\hline GJ393 & M2 & 7.2 & 47 & 1.5 & 1.51 & $-0.50 \pm 0.33$ & -1.50 & $<1.6$ \\
\hline GJ849 & M3.5 & 8.8 & -45 & 0.5 & 2.71 & $0.66 \pm 0.89$ & 0.74 & $<2.9$ \\
\hline GJ791.2 & M4.5 & 8.9 & -17 & 1.0 & 1.70 & $-0.87 \pm 0.57$ & -1.53 & $<1.8$ \\
\hline GJ856A & M3 & 16.0 & -20 & 1.1 & 1.50 & $-0.56 \pm 1.50$ & -0.37 & $<1.6$ \\
\hline GJ9809 & M0 & 24.0 & 03 & 0.5 & 2.20 & $0.36 \pm 2.20$ & 0.16 & $<2.4$ \\
\hline
\end{tabular}

(1) rms estimated for $r<60^{\prime \prime}$ in the map; (2) mean brightness and uncertainty of mean computed by averaging intensities over an effective disk of radius $60 \mathrm{AU}$; (3) the $3 \sigma$ flux density upper limit is computed also over the same effective area (radius = $60 \mathrm{AU}$ ).

Table 5. SCUBA observations of the M-dwarfs associated with moving groups in survey I (Lestrade et al. 2006).

\begin{tabular}{|c|c|c|c|c|c|}
\hline Star & $\begin{array}{l}\text { Sp. } \\
\text { type }\end{array}$ & $\begin{array}{l}\text { Dist. } \\
\text { (pc) }\end{array}$ & $\begin{array}{l}\text { Integration } \\
\text { time } \\
\text { (h) }\end{array}$ & $\begin{array}{l}\text { Flux density }{ }^{a} \\
850 \mu \mathrm{m} \\
(\mathrm{mJy})\end{array}$ & $\begin{array}{l}\operatorname{Size}^{b} \\
(\mathrm{AU})\end{array}$ \\
\hline GJ82 & M4 & 12.0 & 2 & $2.0 \pm 1.4$ & 84 \\
\hline GJ212 & M0.5 & 12.5 & 2 & $1.3 \pm 1.4$ & 88 \\
\hline GJ285 & M4.5 & 5.9 & 1 & $-0.7 \pm 1.9$ & 41 \\
\hline $\mathrm{GJ} 393^{c}$ & $\mathrm{M} 2$ & 7.2 & 1 & $1.9 \pm 1.9$ & 50 \\
\hline GJ507.1 & M1.5 & 17.4 & 1 & $-0.4 \pm 2.0$ & 121 \\
\hline GJ696 & M0 & 21.9 & 2 & $0.8 \pm 0.8$ & 150 \\
\hline GJ9809 ${ }^{c}$ & M0 & 24.9 & 1 & $-5.2 \pm 2.3$ & 174 \\
\hline GJ4247 ${ }^{c}$ & M4 & 9.0 & 1 & $1.1 \pm 2.1$ & 63 \\
\hline GJ277B & M3.5 & 11.5 & 1 & $-2.1 \pm 1.8$ & 80 \\
\hline GJ842.2 & M0.5 & 20.9 & 4 & $25.0 \pm 4.6$ & 300 \\
\hline GJ890 & M2 & 21.9 & 2 & $-2.6 \pm 1.6$ & 153 \\
\hline GJ875.1 & M3 & 14.2 & 1 & $0.52 \pm 2.1$ & 99 \\
\hline
\end{tabular}

${ }^{a}$ Flux density from wide photometry. ${ }^{b}$ Size is the radius of a debris disk as large as the telescope beam $\left(14^{\prime \prime}\right) .{ }^{c}$ Stars also observed by MAMBO2 in Tables 3 and 4.

optical interferometer CHARA (Berger et al. 2006) is about $2 \sigma$ larger than the ZAMS diameter predicted by Chabrier \& Baraffe (1997) and Siess et al. (2000). If this deviation of only $2 \sigma$ is real, it is an indication of youth instead for GJ526, and this would pose a problem for the Kenyon and Bromley model applied to the hypothetical debris disk around GJ526. We note that, with the dust surface density profile $15 \Sigma_{M M S N} \times\left(r / r_{0}\right)^{-3 / 2}$, the mass of solids in a ring at $r=500 \mathrm{AU}$ and $0.1 r$ in width is $1 M_{\oplus}$, which is enough to form planetesimals. Finally, the dust mass corresponding to the emission of the 5 sources is between $\sim 6$ and $\sim 10$ lunar masses as derived in the Appendix for dust grains with a Dohnanyi size distribution and heated both by the stellar luminosity and the interstellar radiation field. The dust mass is $\sim 22$ lunar masses if derived conventionally for grey body dust with a mass opacity of $1.7 \mathrm{~cm}^{2} \mathrm{~g}^{-1}$ at $850 \mu \mathrm{m}$ and $\propto \lambda^{-1}$ for $\lambda>210 \mu \mathrm{m}$, and with the single dust temperature $4.9 \mathrm{~K}$ at large radius from the $\operatorname{star}(r=500 \mathrm{AU})$ where the interstellar radiation field dominates the heating process of grains (see Appendix).

\subsection{Deep search for debris disks in the MAMBO-2 maps}

We searched for faint debris disks in each MAMBO-2 map by averaging intensities over a disk of increasing radius out to $30^{\prime \prime}$ to see whether or not the mean brightness peaks at some angular radius $\theta$. The radius limit of $30^{\prime \prime}$ comes from the shift-and-add reduction method and the wobbler throw of $60^{\prime \prime}$ used for the observations. The intention with this averaging was to identify a disk whose structure is not directly apparent in the map but whose mean brightness is statistically significant. Naturally, no information about its structure can be recovered with this procedure. The optimum sensitivity of this method is for face-on disks, while highly inclined disks might escape detection. This method is similar to the one used to determine extension limits of debris disks in the mid-IR surveys of Sun-like stars conducted by Smith et al. (2008), although, we do not have the complication of having to accurately substract the photosphere at $\lambda=1.2 \mathrm{~mm}$. Following this procedure, we plotted the mean brightness as a function of angular radius $\theta$ for each star presented in Tables 3 and 4. All the curves were inspected and found to wander around zero mean with excursions $\leq 3 \sigma$ for $\theta$ comprised between $11^{\prime \prime} / 2$ and $30^{\prime \prime}$, indicating that no disk was found by this method.

In Fig. 4, we provide the distribution of the mean brightness $\mu /$ mean brightness uncertainty $\sigma_{\mu}$ listed in Tables 3 and 4 and computed for $\theta$ corresponding specificly to the adopted disk radius $60 \mathrm{AU}$ at the distances of the stars. The corresponding Gaussian probability density function is also plotted in Fig. 4. Comparison between the two distributions indicates there are more high positive and negative ratios $\mu / \sigma_{\mu}$ than expected, although they stay within $\pm 3 \sigma$. It means that there are still some systematic errors in the maps but at a low level, likely caused by remaining atmospheric fluctuations.

\subsection{Upper limits to both fractional dust luminosities and dust masses of the debris disks}

The fractional dust luminosity is the fraction of the stellar radiation absorbed and reprocessed to the infrared and (sub)mm by the dust grains; it is proportional to the fraction of the sky covered by dust as seen from the star (Dominik \& Decin 2003). We used the Stefan-Boltzmann law - black body emission to estimate dust luminosity, and modified it by emissivity $1 / X_{\lambda}$, 


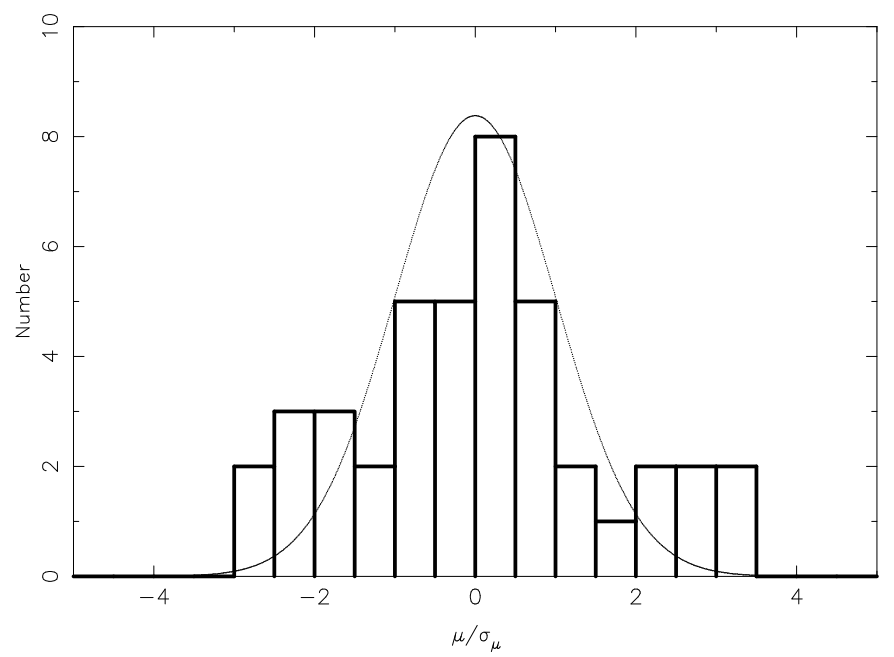

Fig. 4. Distribution of the ratios mean brightness $\mu /$ uncertainty of mean $\sigma_{\mu}$ for the $42 \mathrm{M}$-dwarfs observed with MAMBO-2, i.e., $\mu / \sigma_{\mu}$ listed in Tables 3 and 4 . Here, the mean brightness $\mu$ is computed over a disk of radius 60 AU adopted for each star. The Gaussian probability density function superimposed is scaled so that its integral is 42. Comparison between the two distributions indicates there are more high positive and negative ratios $\mu / \sigma_{\mu}$ than expected, although they stay within $\pm 3 \sigma$. It means that there are still some systematic errors in the maps but at a low level, probably caused by remaining atmospheric fluctuations. No mean brightness is retained as statistically significant in the sample.

with $X_{\lambda}=1$ for $\lambda<210 \mu \mathrm{m}$, and $X_{\lambda}=\lambda / 210$ for $\lambda>210 \mu \mathrm{m}$ (Wyatt 2008). In these conditions, the fractional dust luminosity is

$\frac{L_{\text {dust }}}{L_{*}}=\frac{9 \times 10^{6} c^{2}}{2 \pi h v^{3}}\left(\mathrm{e}^{h v / k T_{\mathrm{d}}}-1\right) \frac{S_{\theta} d^{2} T_{\mathrm{d}}^{4}}{R_{*}^{2} T_{*}^{4}} \times X_{\lambda}$,

the normalization coefficient is such that the measured flux density $S_{\theta}$ is in Jy, the star distance $d$ in pc, the stellar radius $R_{*}$ in $\mathrm{m}$, the dust and stellar effective temperatures $T_{\mathrm{d}}$ and $T_{*}$ in $\mathrm{K}$, the Planck and Boltzmann constants $h$ and $k$ in $\mathrm{J} \times \mathrm{s}$ and $\mathrm{J} / \mathrm{K}$, the speed of light $c$ in $\mathrm{m} / \mathrm{s}$, and the frequency of observation $v$ in $\mathrm{Hz}$.

The standard argument used to fix dust temperature $T_{\mathrm{d}}$ in mid-IR surveys of debris disks is that observations are most sensitive to the dust emission peak, and $T_{\mathrm{d}}$ is derived from the Wien law. In the (sub)mm range, this law is inappropriate for $T_{\mathrm{d}}$ since we observe in the Rayleigh-Jeans limit. To keep full generality, we avoid choosing a dust temperature $T_{\mathrm{d}}$ at some arbitrary radius but plot in Figs. 5 and 6 fractional dust luminosities and dust masses as functions of disk radius $r$ between 1 and $1000 \mathrm{AU}$ following the approach by Bryden et al. (2006) and Wyatt (2008). At disk radius $r$, we use the dust temperature

$T_{\mathrm{d}}=278 \times\left(L_{*}^{0.25}\right) \times\left(r^{-0.5}\right)$,

from Black Body equilibrium, where $L_{*}$ is the stellar luminosity in $L_{\odot}$ and $r$ is in AU (Backman \& Paresce 1993). We note that by combining Eqs. (1) and (2), one derives the expression for fractional dust luminosity given by Eq. (8) of Wyatt (2008), where the stellar luminosity $L_{*}$ cancels out.

We adopt the $3 \sigma$ flux density limit for $S_{\theta}$ in Eq. (1) by integrating brightness over a face-on disk of radius $r$, or $\theta$ at the distance of the star. If $2 \theta>11^{\prime \prime}$ (i.e., >IRAM $30 \mathrm{~m}$ beam):

$S_{\theta}=3 \times \mathrm{rms} \times\left(2 \theta^{\prime \prime} / 11^{\prime \prime}\right) \quad(\mathrm{mJy})$.

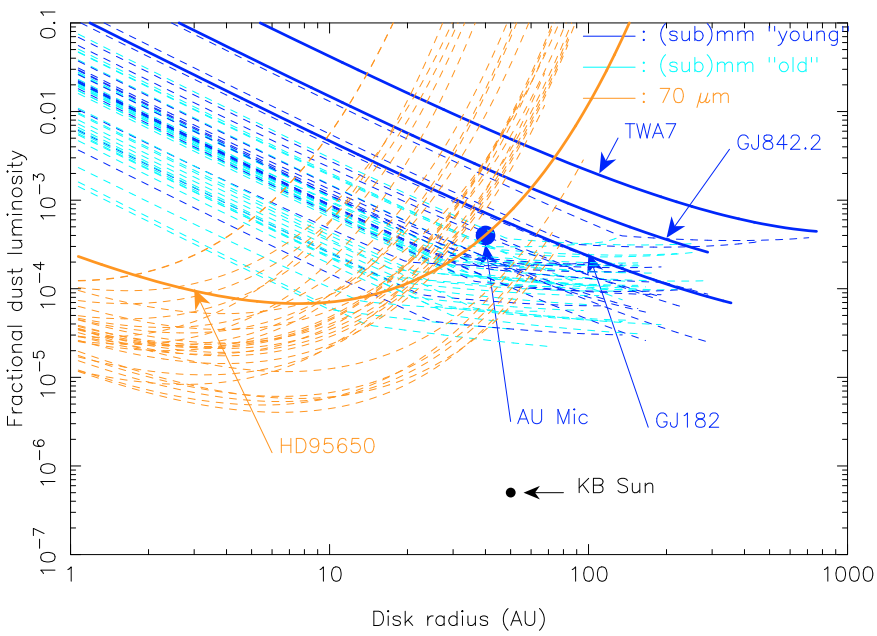

Fig. 5. Constraints on the dust luminosity fraction versus disk radius. Note that the $x$-axis shows the single radius corresponding to the single temperature of a disk (ring) assumed infinitely narrow in our model. We consider the wide range of radii from 1 to $1000 \mathrm{AU}$ as plausible for rings of debris. Upper limits are shown as dashed lines, detections as solid lines. Blue lines correspond to the (sub)millimeter observations: i.e., our sample of $50 \mathrm{M}$-dwarfs for which dark blue is used for "young" M-dwarfs (ages <200 Myr) and light blue for "old" M-dwarfs (likely a few Gyr); the submm disk detected around AU Mic and resolved (Liu et al. 2004; Liu 2004) is marked by a single dot; the two submm disks detected but not clearly resolved around GJ182 (Liu et al. 2004) and around GJ842.2 (Lestrade et al. 2006) are marked as curves; and the submm transition disk detected around the pre-main sequence M 1 dwarf TWA7 (Matthews et al. 2007) is also included. Orange lines correspond to Spitzer $70 \mu \mathrm{m}$ observations: i.e., the sample of $41 \mathrm{M}$-dwarfs of Gautier et al. (2007) and the detection of the M 0 dwarf HD95650 (Smith et al. 2006). There are $16 \mathrm{M}$-dwarfs in common between the two samples. These two sets of data are complementary to constrain the existence of warm dust around M-dwarfs at moderate radii ( $<20 \mathrm{AU})$ and cold dust at large radii $(>20 \mathrm{AU})$. Disk diameters probed by the observations are limited by the angular size of $60^{\prime \prime}$ for the MAMBO-2 maps, of $28^{\prime \prime}$ for SCUBA wide photometry, and of 38.4" for Spitzer aperture photometry (Gautier et al. 2007). Some curves are terminated at less than $1000 \mathrm{AU}$ because of these angular limits. The calculation of the fractional dust luminosity is described in the text. (This figure is available in color in electronic form.)

This formula takes into account that the mean brightness uncer-

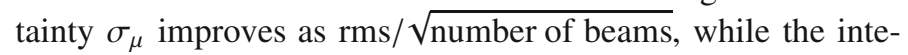
grated flux density increases as the number of beams of the disk area. Now if $2 \theta<11^{\prime \prime}$

$S_{\theta}=3 \times \mathrm{rms} \quad(\mathrm{mJy})$.

We used the rms of Tables 3 and 4 that correspond to the central part of the maps, thus underestimating slightly the upper limits computed.

In Fig. 5, we show the resulting fractional dust luminosity upper limits to $r$ between 1 and 1000 AU. These functions first show a steep negative slope for $2 \theta<11^{\prime \prime}$ making $S_{\theta}$ constant in Eq. (1), then these functions level off when $S_{\theta}$ linearly increases with $\theta$, and finally they increase when the dust temperature saturates at $4.9 \mathrm{~K}$ because the interstellar radiation field becomes dominant over the stellar field (see Appendix). In this figure, we have added the upper limits to the fractional dust luminosities for the $41 \mathrm{M}$-dwarfs observed at $70 \mu \mathrm{m}$ by Spitzer (Gautier et al. 2007), computed in a similar fashion for their $3 \sigma$ flux densities. The figure shows that the two sets of data are complementary, and lead to a uniform fractional dust luminosity over a large extent of disk radii for the dozen of M-dwarfs common to the two 


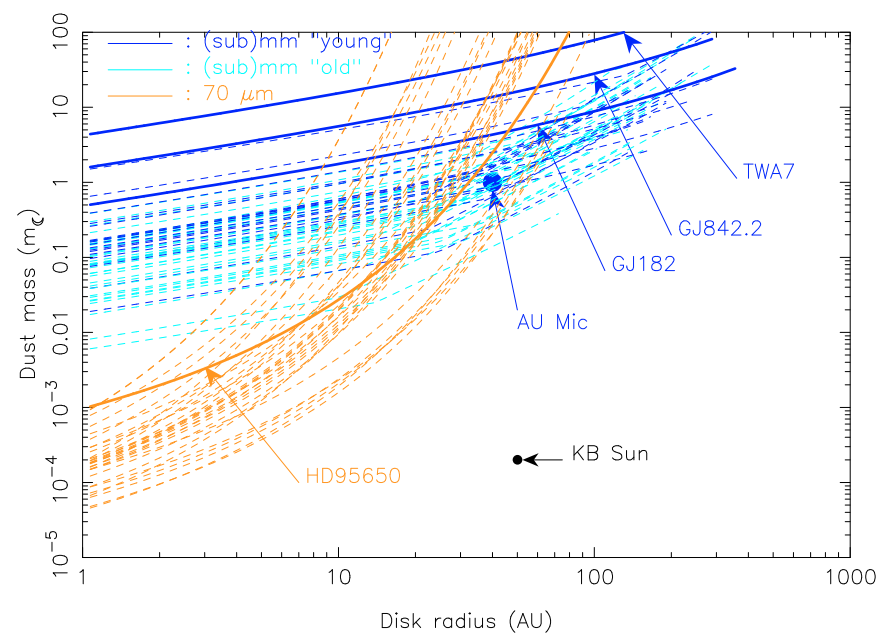

Fig. 6. Constraints on the dust mass versus disk radius. Note that the $x$-axis shows the single radius corresponding to the single temperature of a disk (ring) assumed infinitely narrow in our model. We consider the wide range of radii from 1 to $1000 \mathrm{AU}$ as plausible for rings of debris. Upper limits are shown as dashed lines, detections as solid lines. Blue lines correspond to the (sub)millimeter observations and orange lines to the Spitzer $70 \mu \mathrm{m}$ observations as described in the legend of Fig. 5. The mass opacity used to convert flux density to dust mass with the standard optically thin emission model is $\kappa=1.7 \mathrm{~cm}^{2} \mathrm{~g}^{-1}$ at $850 \mu \mathrm{m}$ and $\propto \lambda^{-1}$ for $\lambda>210 \mu \mathrm{m}$. Additional information are in the text. (This figure is available in color in electronic form.)

data sets. In Fig. 6, we present the corresponding dust masses as a function of $r$ for both samples computed with the optically thin emission model (e.g., Zuckerman 2001) and the mass opacity $\kappa_{\lambda} \propto \lambda^{-1}$ for $\lambda>210 \mu \mathrm{m}$ and $\kappa_{850 \mu \mathrm{m}}=1.7 \mathrm{~cm}^{2} \mathrm{~g}^{-1}$.

\section{Discussion}

The single cold debris disk found in our surveys (GJ842.2, see details in Lestrade et al. (2006), and excluding GJ526 at this stage) makes the detection fraction to be $2_{-1.5}^{+4.5} \%$ in our sample of $50 \mathrm{M}$-dwarfs. The limits are based on the Binomial distribution for a small number sample and are such that $68 \%$ of the probability is between the lower and upper uncertainties, and the peak probablity is the observed fraction 1/50, following Burgasser et al. (2003). We recall that Gautier et al. (2007) had no detection in a sample of 62 nearby M-dwarfs at $24 \mu \mathrm{m}$ and no detection in a subsample of 41 of them at $70 \mu \mathrm{m}$, i.e., rate $<7 \%$. In a different way, Forbrich et al. (2008) detected photometric excesses at $24 \mu \mathrm{m}$ tracing warm dust around $11 \mathrm{M}$-dwarfs in the young open cluster NGC2457 (20-40 Myr) representing $4.9_{-1.8}^{+1.8} \%$ of the 225 highly probable member M-stars identified within it.

To discuss how detectability of cold debris disks depends on the mass of the central star, we compared our result with the observed fractions of cold debris disks around stars more massive than M-dwarfs. In the literature, we find $\sim 30$ cold debris disks around A-to-K type stars detected by submm observations (see Fig. 3 and caption of Wyatt 2008). However most of these detections are from JCMT/SCUBA surveys of IRAS biased samples, i.e., targets with prior IRAS excess detections, unlike our M-dwarfs, for which no such prior knowledge was used in the selection of the sample. There are only two submm surveys of A-to-K type stars that are unbiased in this respect and have depths of $\sim 1-3 \mathrm{mJy}$ at $850 \mu \mathrm{m}$, comparable to our sensitivity at $1.2 \mathrm{~mm}$ : the JCMT survey by Najita \& Williams (2005) of thirteen F5-to-K3 stars (10 Myr < ages < $180 \mathrm{Myr}$,

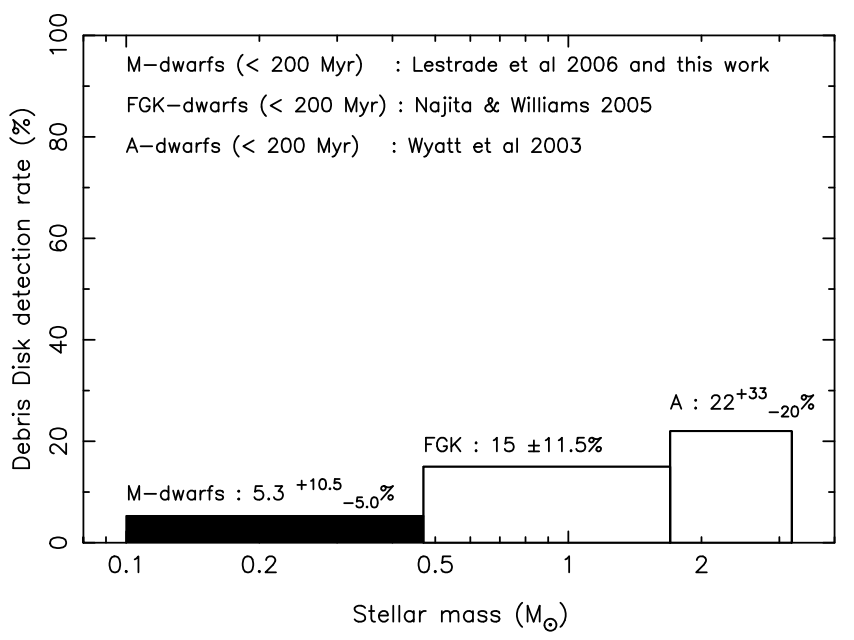

Fig. 7. Detection rates of cold debris disks versus stellar masses (stellar types) for stars younger than 200 Myr. These rates and uncertainties are for disks having dust fractional luminosities larger than the limits shown in Fig. 8 of the (sub)mm surveys used.

$10<d<78$ pc, 3 detections) and the JCMT survey by Wyatt et al. (2003) of nine B7-to-A0 stars $(86<d<938$ pc, 2 detections) and of thirteen F3-to-K5 stars $(27<d<250$ pc, 1 detection) that are all part of Lindroos binaries (14 Myr $<$ ages $<170 \mathrm{Myr}$ ). Combining these two submm surveys, we determine the cold disk fractions of $22_{-20}^{+33} \%$ for young A stars ( 2 detections/9) and of $15_{-11.5}^{+11.5} \%$ for young FGK stars (4/26). For a meaningful comparison, instructed by Spitzer surveys where the age factor is crucial for detectability of warm debris disks, we selected the 19 youngest $M$ dwarfs with ages between 20 and $200 \mathrm{Myr}$ in our sample. The disk fraction for these "young" Mdwarfs is $5.3_{-5.0}^{+10.5 \%}$. Therefore, for this age range, there is an apparent trend in these three fractions, indicative of fewer cold disks detected for later stellar types - lower star masses - although at a low statistical significance (Fig. 7). Nonetheless, this trend is notable because the surveys are deeper for later stellar types as shown in Fig. 8, for disk radii $<100$ AU. Interestingly, this trend has also been found at $70 \mu \mathrm{m}$ in a sample of $\mathrm{A}$ to $\mathrm{M}$ stars with ages between $8 \mathrm{Myr}$ and $1 \mathrm{Gyr}$ by Plavchan et al. (2009).

We also determine the cold disk fraction of $<10 \%$ for the "old" M-dwarfs of our sample with undetermined ages but that are probably as old as average Galactic disk stars $(8.8 \pm 1.7 \mathrm{Gyr}$, del Peloso et al. 2005). This may indicate that the cold disk fraction decreases with stellar age, in a similar way to warm disks (Rieke et al. 2005). We note that the "old" M-dwarfs were observed with higher sensitivity as seen in Figs. 5 and 6, where "young" and "old" M-dwarfs have been color-coded in dark and light blue. The higher sensitivity to the "old" M-dwarfs is because they are nearer than the "young" M-dwarfs.

We now examine possible reasons why M-dwarfs have less detectable disks.

Theoretical models of star formation predict that protoplanetary disks are less massive around M-dwarfs than around higher mass stars. Vorobyov \& Basu (2008) predict $\left\langle M_{\mathrm{d}}\right\rangle \propto\left\langle M_{*}\right\rangle^{1.3 \pm 0.1}$, where disk mass $\left\langle M_{\mathrm{d}}\right\rangle$ and star mass $\left\langle M_{*}\right\rangle$ are time-averaged over the star accretion period ( 0.5 to $2.5 \mathrm{Myr}$ ). Consequently, less primordial materials could limit planet formation around M-dwarfs. Collecting masses of protoplanetary disks determined by (sub)mm observations in the nearest star forming regions, Natta et al. (2000) found $M_{\mathrm{d}} \propto M_{*}^{0.64 \pm 0.14}$, but 


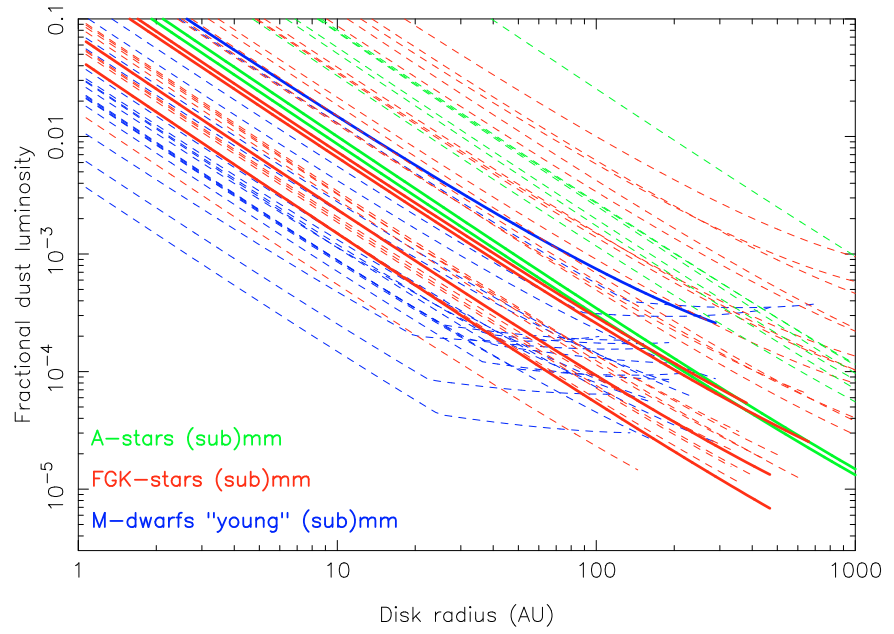

Fig. 8. Dust luminosity versus disk radius limits of the three (sub)mm surveys used to determine the cold disk fractions of "young" M-, FGKand A-type stars. Shown are the dust luminosity fractions of the 19 youngest M-dwarfs of our sample (blue), of the 9 young A-stars (green), and of the 26 young FGK-stars (red) in the two $850 \mu \mathrm{m}$ surveys of Wyatt et al. (2003) and of Najita \& Williams (2005). All these stars are less than 200 Myr old. Most of the curves are upper limits (dashed lines). The 7 detections are marked by full lines. This plot emphasizes that for stars of lower masses (later stellar types), even though the surveys are more sensitive, fewer disks are detected. Note that the $x$-axis shows the single radius corresponding to the single temperature of a disk (ring) assumed infinitely narrow in our model. We consider the wide range of radii from 1 to $1000 \mathrm{AU}$ as plausible for rings of debris. (This figure is available in color in electronic form.)

Andrews \& Williams $(2005,2007)$ show that $M_{\mathrm{d}}$ versus $M_{*}$ in Taurus-Auriga is so widely scattered between 0.001 and $0.2 M_{\odot}$ that it precludes any meaningful correlation fit. The comparison of the disk masses of 6 members of the nearby young TW Hydrae Association (TWA) also suggests no correlation between disk masses and stellar types for these reasonably coeval disks (Matthews et al. 2007).

Removal of circumstellar dust by the Poynting-Robertson effect and radiation pressure processes are diminished around $\mathrm{M}$ dwarfs because they are less luminous than solar-type stars, and so dust generated by collisions in any remnant planetesimal belt should remain there longer, giving rise to detectable emission. However, the opposite conclusion has been reached by Plavchan et al. (2005), highlighting the fact that dust removal around $\mathrm{M}$ dwarfs could be dominated by the drag caused by strong winds associated with their high coronal and chromospheric activities.

The formation of planetesimals and planets depends on the timescales between the competing processes of coagulation and evaporation in the early period of accumulation. Theory predicts that $t_{\text {coag }}$ increases and $t_{\text {evap }}$ decreases with both the central star mass and the strength of the FUV and EUV radiation field (Eq. (47) and (48) in Adams et al. 2004, respectively). From their Fig. 10, it can be seen that a time longer than $10 \mathrm{Myr}$ is required to evaporate a protoplanetary disk around a solar-mass star, whereas only a few Myr are required to evaporate the same disk around a low-mass M-dwarf in a stellar cluster with a moderate UV flux of $\sim 3000 G_{0}$. This might quench planet formation around M-dwarfs.

Early stripping of planetesimals by passing stars is likely since most stars are born in clusters where stellar encounters as close as $160 \mathrm{AU}$ are likely in the first $100 \mathrm{Myr}$ (Kenyon \& Bromley 2004b). The disruption of planetesimal disks by close stellar encounters was studied for the A6 star $\beta$ Pic by Larwood $\&$ Kalas (2001). Depending on both the passing star's trajectory and the relative star masses, they found that $1 \%$ to $48 \%$ of the planetesimals are lost after encounters. Related issues for planetary systems were also discussed by Malmberg et al. (2007).

A lack of gaseous giant planets around M-dwarfs is predicted by Laughlin et al. (2004), because of the longer duration required to build a core of $15 M_{\oplus}$. This lack of giant planets might reduce the production of second generation dust in M-dwarf debris disks because of weaker gravitationnal stirring (Wilner et al. 2002), diminishing their detectability.

This series of arguments leads to the expectation that debris disks around M-dwarfs might be intrinsically less dusty and therefore more difficult to detect than those around more massive stars of the same age. However, most of these arguments assume that the initial protoplanetary disk mass scales with the central star mass, which may be plausible but is observationally not well established.

\section{Conclusion}

To search for emission from cold debris disks, we have used the MAMBO-2 bolometer camera at the IRAM $30 \mathrm{~m}$ telescope to map 42 nearby M-dwarfs at $\lambda=1.2 \mathrm{~mm}$ to a noise level of between 0.6 and $2.8 \mathrm{mJy}$ per $11^{\prime \prime}$ beam. We also reanalyzed our earlier MAMBO-2 and SCUBA data to form a coherent sample of 50 M-dwarfs. Only one cold debris disk was detected, surrounding the M 0.5 dwarf GJ842.2. To discuss how the detectability of cold debris disks depends on the mass of the central star, we have compared this result to the observed fractions of cold disks for more massive stars in the two submm surveys of Wyatt et al. (2003) and Najita \& Williams (2005), who report detection rates of $22_{-20}^{+33} \%$ for A-stars and $15_{-11.5}^{+11.5} \%$ for FGK-stars with stellar ages between 10 and $180 \mathrm{Myr}$. For the 19 youngest M-dwarfs ( $\leq 200 \mathrm{Myr}$ ) of our sample, we found a detection rate of $5.3_{-5.0}^{+10.5} \%$. Hence, for this age range, there is a mild trend in these three detections rates, indicative of fewer cold debris disks detected for later stellar types - lower star masses - although at a low statistical significance. Nonethless this trend is notable because the sensitivities of these surveys are deeper for later stellar types. We also determine the cold disk fraction of $<10 \%$ for the "old" M-dwarfs (likely to be a few Gyr) of our sample, indicative of the cold disk fraction decreasing with stellar age, as is also seen for warm disks. Future observations of a larger and more tightly controlled sample of stars of all stellar types with Herschel in the far-IR and deeper observations in the (sub)mm will help to clarify these issues.

\section{Appendix A: Computation of the dust temperature and mass of the possible large debris disk around GJ526}

The source that heats dust in debris disks is usually the stellar radiation field, but in a large debris disk, as possibly found around GJ526, the interstellar radiation field dominates at some radius from the star. We compute this transition radius for several stellar spectral types by numerically solving the integral equation of a grain at thermal equilibrium absorbing both the stellar and interstellar incident fields

$$
\begin{aligned}
\int_{0}^{+\infty} Q_{\mathrm{abs}}(\lambda, a) \cdot\left(\pi a^{2} J_{*}(\lambda, r)+4 \pi a^{2} J_{\mathrm{ISRF}}(\lambda)\right) \mathrm{d} \lambda= \\
\int_{0}^{+\infty} 4 \pi a^{2} Q_{\mathrm{abs}}(\lambda, a) \pi B\left(\lambda, T_{\mathrm{g}}\right) \mathrm{d} \lambda,(\mathrm{A} .1)
\end{aligned}
$$




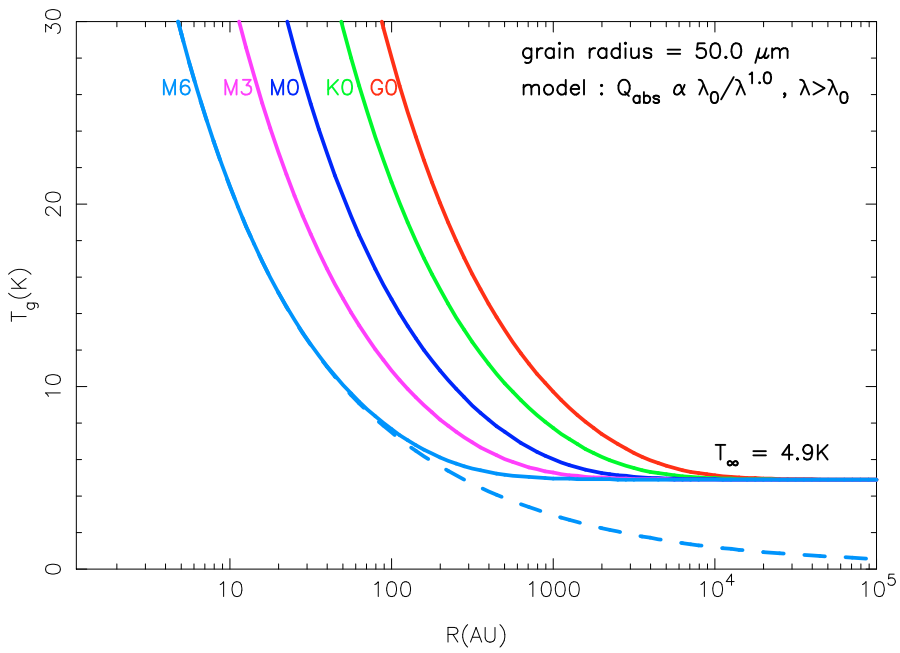

Fig. A.1. Variation in the temperature of a grain $100 \mu \mathrm{m}$ in diameter exposed to both the radiation of the stellar field and the isotropic interstellar radiation field. This latter field dominates at some disk radius $R$ that depends on the stellar luminosity. The saturation temperation of $4.9 \mathrm{~K}$ due to the interstellar field is consistent with the computation of Krügel (2003, p. 249). For M 6 only, the dashed line indicates the grain temperature when the interstellar radiation field is not included. (This figure is available in color in electronic form.)

where $Q_{\mathrm{abs}}(\lambda, a)=1$ for $\lambda<\lambda_{0}$ and $Q_{\mathrm{abs}}(\lambda, a)=\lambda_{0} / \lambda$ with $\lambda_{0}=$ $2 \pi a$ for $\lambda>\lambda_{0}$, which approximate the absorption efficiency computed for carbon by Laor \& Draine (1993). The parameter $a$ is the radius of a spherical grain. The expression $B\left(\lambda, T_{\mathrm{g}}\right)$ is the Planck function at grain temperature $T_{\mathrm{g}}$ that depends on $a$. The intensity of the stellar radiation field $J_{*}(\lambda, r)$ is $\pi B\left(T_{*}, \lambda\right) \times$ $\left(4 \pi R_{*} / 4 \pi r\right)^{2}$ at disk radius $r$, where the star is characterized by its effective temperature $T_{*}$ and its radius $R_{*}$. The intensity of the interstellar radiation field $J_{\mathrm{ISRF}}(\lambda)$ in the solar neighbourhood is

$J_{\operatorname{ISRF}}(\lambda)=\sum_{i=0}^{i=6} C_{i} \pi B\left(\lambda, T_{i}\right)$

with components $C_{0}=1, T_{0}=2.7 \mathrm{~K}, C_{1}=4 \times 10^{-17}, T_{1}=$ $27000 \mathrm{~K}, C_{2}=10^{-14}, T_{2}=7500 \mathrm{~K}, C_{3}=10^{-13}, T_{3}=$ $4000 \mathrm{~K}, C_{4}=4 \times 10^{-13}, T_{4}=3000 \mathrm{~K}, C_{5}=1.5 \times 10^{-10}, T_{5}=$ $400 \mathrm{~K}, C_{6}=0.5 \times 10^{-5}$, and $T_{6}=46 \mathrm{~K}$, at galactocentric distance $10 \mathrm{kpc}$ (Mathis et al. 1983). In Fig. A.1, we solved Eq. (A1) numerically to determine dust temperature $T_{\mathrm{g}}(r)$ as a function of disk radius in modelling grains with the single size $2 a=100 \mu \mathrm{m}$, typical of submm observations, and for several stellar spectral types. For GJ526 (M 1.5), this figure shows that the interstellar radiation field becomes dominant at $r>1000 \mathrm{AU}$, but this transition radius is only 200 AU for spectral type M 6.

In Fig. A.2, we use a more realistic model for the dust in adopting the standard grain size distribution $\mathrm{d} N=N_{0} a^{-3.5} \mathrm{~d} a$ (Dohnanyi 1969) to compute temperature $T_{\mathrm{g}}(a)$ as a function of grain size at $r=500 \mathrm{AU}$ with Eq. (A1) but modified to include the grain size distribution. Temperature increases significantly for $a<10 \mu \mathrm{m}$, which is relevant for M-dwarfs because the grain blow-out size is small. For GJ526 $\left(0.031 L_{\odot}\right)$, it is as small as $0.04 \mu \mathrm{m}$ for carboneous grains, ignoring the effect of stellar wind drag for this star, which has a low coronal/chromospheric activity $\left(\log L_{\mathrm{x}}=26.87\left[10^{-7} \mathrm{~W}\right]\right.$, Schmitt \& Liefke, 2004). Finally, we use this temperature function $T_{\mathrm{g}}(a)$ at $r=500 \mathrm{AU}$ to determine the dust mass around GJ526 by matching the total flux density of the 5 clumps $\left(S_{1.2 \mathrm{~mm}}=21.2 \pm 2 \mathrm{mJy}\right)$ to the flux

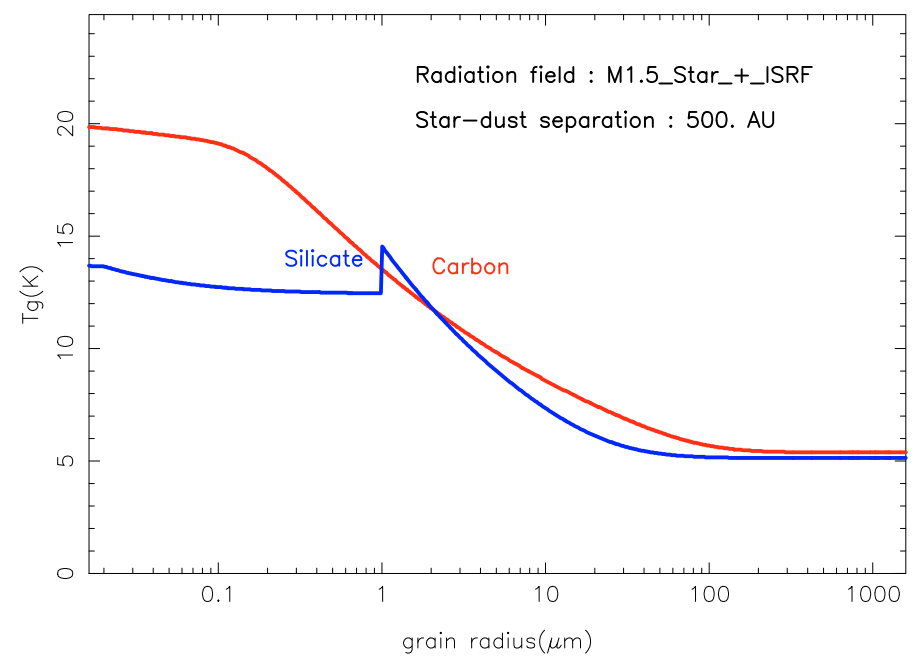

Fig. A.2. Variation in temperature as a function of grain radius at $500 \mathrm{AU}$ from the M 1.5 dwarf GJ526. Similar results are reported by Krügel (2003, p. 249). The discontinuity in the silicate curve arises from the piecewise-defined function $Q_{\mathrm{abs}}(\lambda, a)$ adopted from Laor \& Draine (1993). (This figure is available in color in electronic form.)

density of our model. If $d$ is the distance to the star, the predicted flux density is

$S_{v}=\frac{N_{0}}{4 \pi d^{2}} \int_{a_{\min }}^{a_{\max }} 4 \pi a^{2} Q_{\mathrm{abs}}(v, a) \cdot \pi B\left(v, T_{\mathrm{g}}(a)\right) \cdot a^{-3.5} \mathrm{~d} a$

where the limit $a_{\min }$ is set by the blow-out size ( $a_{\min }=0.04 \mu \mathrm{m}$ for GJ526). The limit $a_{\max }$ is related to the total dust mass $M_{\mathrm{d}}=\frac{8}{3} \pi \rho N_{0} \sqrt{a_{\max }}$ for the size distribution above and spherical grains of density $\rho$. The dust mass $M_{\mathrm{d}}$ is probed by the measured flux density at the observing $\lambda$. Practically, the grain size limit $a_{\max }$ is the value corresponding to convergence of the computation of the power emitted by $M_{\mathrm{d}}$ over a band $2 b$ centered on the observed wavelength $(1.2 \mathrm{~mm})$. We adopted the convergence criterium of $5 \%$ to match the relative accuracy of the measured flux density. In other words, $a_{\max }$ is increased until the integral below converges to within 5\%:

$\int_{+1.2 m m-b}^{+1.2 m m+b} \int_{a_{\min }}^{a_{\max }} 4 \pi a^{2} Q_{\mathrm{abs}}(\lambda, a) \cdot \pi B\left(\lambda, T_{\mathrm{g}}(a)\right) \cdot a^{-3.5} \mathrm{~d} a \mathrm{~d} \lambda .(\mathrm{A} .3)$

This power would need to be computed more accurately by including the small contribution to the emission of larger pebbles only if the flux density were measured more accurately. We found that $a_{\max }$ is $\sim 24 \mathrm{~mm}$ and $\sim 36.4 \mathrm{~mm}$ for carboneous and silicate grains, respectively, and, independently of the choice $b=5 \%, 10 \%, 20 \% \times 1.2 \mathrm{~mm}$. Similar calculation was made by Wyatt \& Dent (2002) for the A3V dwarf Fomalhaut by integrating not only over grain size but also over a range of radius $r$ to account for the spatial distribution of the dust in a ring. They found that $95 \%$ of the flux density is the emission from grains and pebbles smaller than $100 \mathrm{~mm}$ in radius. This is comparable to our determination of $a_{\max }$ and the difference must be caused by the different function for $T_{\mathrm{g}}(a)$ and their hypothesis of spatially distributed dust.

Figures A. 3 and A. 4 show the SED of the dust around GJ526 based on our model of both carboneous and silicate grains adjusted to the flux density measured at $1.2 \mathrm{~mm}$. The dust mass determined by our model is between $\sim 6$ and $\sim 10$ lunar masses. We have added in these figures, the SED based on the standard greybody model of optically thin dust emission (Hildebrand 1983; 


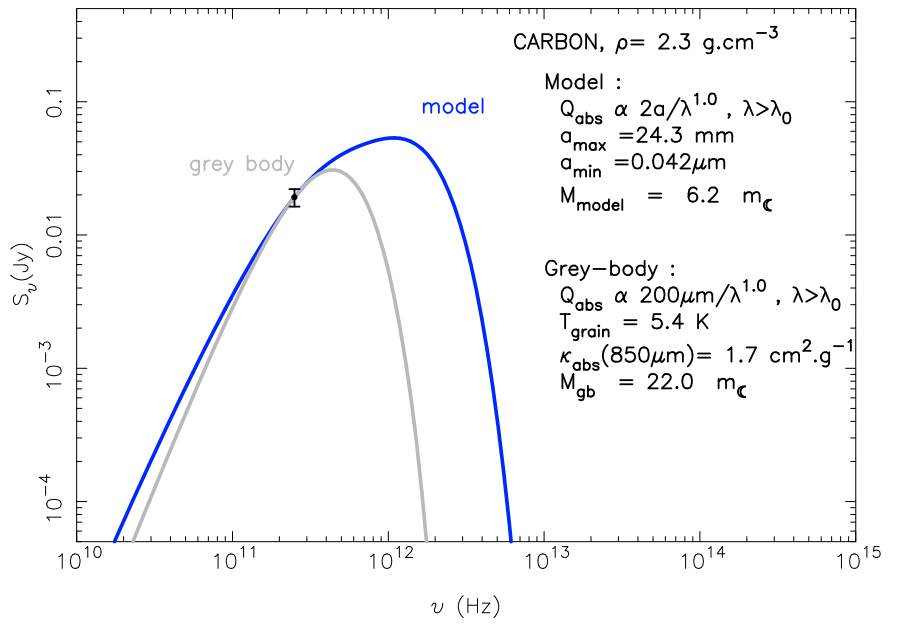

Fig. A.3. SED of carboneous dust around GJ526 based on our model fitted to the $1.2 \mathrm{~mm}$ flux density. Our model includes the collisional dust size distribution $\mathrm{d} N=a^{-3.5} \mathrm{~d} a$ and the resulting non-uniform temperature for grains of various sizes computed in Fig. A.2. Superimposed is the corresponding grey-body model computed with the single grain temperature $4.9 \mathrm{~K}$ and standard mass opacity $1.7 \mathrm{~cm}^{2} \mathrm{~g}^{-1}$ at $\lambda=850 \mu \mathrm{m}$ scaled with the efficiency $210 \mu \mathrm{m} / \lambda$ (see text). (This figure is available in color in electronic form.)

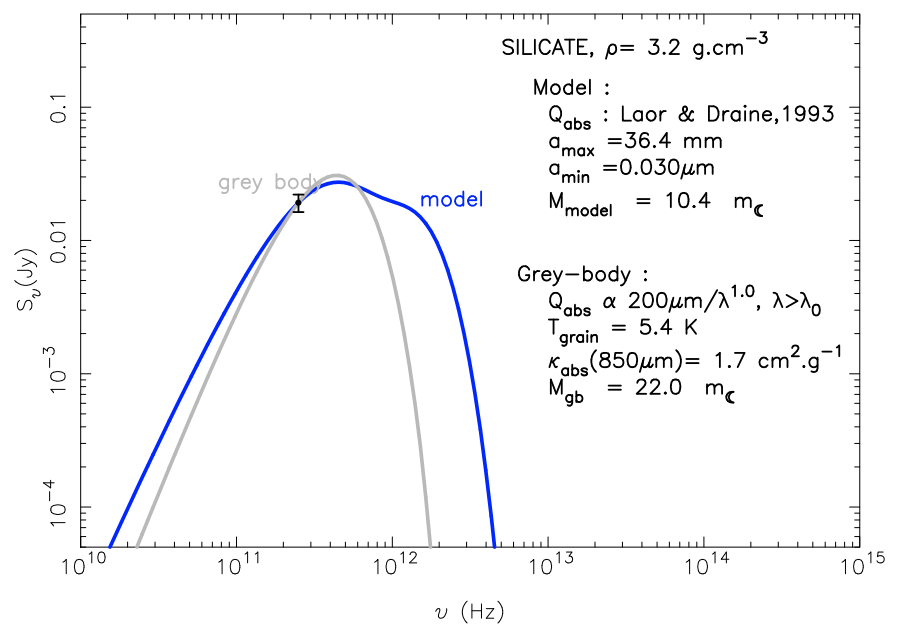

Fig. A.4. SED of silicate dust around GJ526 based on our model and fitted to the $1.2 \mathrm{~mm}$ flux density. Same comments as in Fig. A.3. (This figure is available in color in electronic form.)

and Zuckerman 2001)

$S_{v}=M_{\mathrm{d}} \times B\left(v, T_{\mathrm{g}}\right) \times \kappa_{\mathrm{abs}} / d^{2}$

where the mass opacity $\kappa_{\mathrm{abs}}$ is conventionally taken to be $1.7 \mathrm{~cm}^{2} \mathrm{~g}^{-1}$ at $850 \mu \mathrm{m}$ and is scaled by $210 \mu \mathrm{m} / \lambda$ for $\lambda>$ $210 \mu \mathrm{m}$. This grey dust mass is about 2 to 4 times higher than the one determined by our model. This level of accuracy is typical of current dust mass estimates for debris disks, presently.

The SED of our model is significantly more extended to the far-IR and to the radio than the grey-body model when both models match the flux density at $\lambda=1.2 \mathrm{~mm}$. We point out that this far-IR extension is much less pronounced if our model is applied to an A-star. Future observations with Herschel will test these SED differences and will thus probe, in essence, the collisional dust size distribution adopted and the non-uniform grain temperature.
Acknowledgements. We are grateful to the staff of the IRAM 30-m telescope, especially Dr S. Leon, for his dedication in managing the MAMBO-2 pool during the transistion period with the new contral system and for his unfailing determination to optimize the science return of the telescope despite harsh conditions. We would like to thank an anonymous referee for his careful reading of the manuscript and constructive remarks. This research has made use of the SIMBAD database and of the VizieR catalog access tool, opera ted at the Centre de Données Stellaires (CDS), Strasbourg, France. This publication makes use of data products from the Two Micron All Sky Survey, which is a joint project of the University of Massachusetts and the Infrared Processing and Analysis Center/California Institute of Technology, funded by the National Aeronautics and Space Administration and the National Science Foundation. This publication has made use of data products from the SDSS and SDSS-II funded by the Alfred P. Sloan Foundation, the Participating Institutions, the National Science Foundation, the US Department of Energy, the National Aeronautics and Space Administration, the Japanese Monbukagakusho, the Max Planck Society, and the Higher Education Funding Council for England. This work is based in part on observations made with the Spitzer Space Telescope, which is operated by the Jet Propulsion Laboratory, California Institute of Technology under a contract with NASA.

\section{References}

Adams, F. C., Hollenbach, D., Laughlin, G., \& Gorti, U. 2004, ApJ, 611, 360 Allard, F., Hauschildt, P. H., Alexander, D. R., Tamanai, A., \& Schweitzer, A. 2001, ApJ, 556, 357

Andrews, S. M., \& Williams, J. P. 2005, ApJ, 631, 1134

Andrews, S. M., \& Williams, J. P. 2007, ApJ, 671, 1800

Augereau, J.-C., \& Paploizou, J. C. B. 2004, A\&A, 414, 1153

Aumann, H. H., Beichman, C. A., Gillett, F. C., et al. 1984, ApJ, 278, 23

Backman D. E., \& Paresce, F. 1993, in Protostars and Planets III, ed. E. H. Levy,

\& J. I. Lumine (Tucson: Univ. Press), 1253

Berger D. H., Gies, D. R., McAlister, H. A., et al. 2006, ApJ, 644, 475

Bertoldi, F., Carilli, C., Aravena, M., et al. 2007, ApJS, 172, 132

Bryden, G. C., Beichman, C. A., Trilling, D. E., et al. 2006, ApJ, 636, 1098

Burgasser, A. J., Kirkpatrick, J. D., Reid, I. N., et al. 2003, ApJ, 586, 512

Chabrier, G., \& Baraffe, I. 1997, A\&A, 327, 1039

Condon, J. J., Cotton, W. D., Greisen, E. W., et al. 1998, AJ, 115, 1693

del Peloso, E. F., da Silva, L., Porto de Mello, G. F., \& Arany-Prado, L. I. 2005, A\&A, 440, 1153

Diggle, P. J. 2003, in Statistical Analysis of Spatial Point Patterns (Hodder Arnold)

Dohnanyi, J. S., 1969, J. Geophys. Res., 74, 2531

Dominik, C., \& Decin, G. 2003, ApJ, 583, 626

Dutrey, A., Guilloteau, S., \& Simon, M. 1994, A\&A, 286, 149

Forbrich, J., Lada, C. J., Muench, A. A., \& Teixeira, P. S. 2008, ApJ, 687, 1107

Gautier, T. N., Rieke, G. H., Stansberry, J., et al. 2007, ApJ, 667, 527

Gomes, R., Levison, H. F., Tsiganis, K., \& Morbidelli, A. 2005, Nature, 435, 466.

Greaves, J. S., Holland, W. S., Wyatt, M. C., et al. 2005, ApJ, 619, 187

Greve, T. R., Ivison, R. J., Bertoldi, F., et al. 2004, MNRAS, 354, 779

Hahn, J. M., \& Malhotra, R. 1999, AJ, 117, 3041

Hildebrand, R. H. 1983, QJLR Astr. Soc., 24, 267

Holland, W. S., Greaves, J. S., Zuckerman, B., et al. 1998, Nature, 392, 788

Kalas, P., Graham, J., \& Clampin, M. 2005, Nature, 435, 1067

Kenyon, S. J., \& Bromley, B. C. 2004a, AJ, 127, 513

Kenyon, S. J., \& Bromley, B. C. 2004b, Nature, 432, 598

Kreysa, E., Gemünd, H. P., Gromke, J., et al. 1998, in Advanced Technology MMW, Radio, and Terahertz Telescopes, ed. T. G. Phillips, SPIE, 3357, 319

Krist, J. E., Ardila, D. R., Golimowski, D. A., et al. 2005, AJ, 129, 1008

Krügel, E. 2003, in The Physics of Interstellar Dust (Institute of Physics Publishing: Series Astronomy \& Astrophysics)

Laor, A., \& Draine, B. T. 1993, ApJ, 402, 441

Larwood, J. D., \& Kalas, P. G. 2001, MNRAS, 323, 402

Laughlin, G., Bodenheimer, P., \& Adams, F. 2004, ApJ, 612, L73

Lestrade, J.-F., Wyatt, M. C., Bertoldi, F., Dent, W. R. F., \& Menten, K. M. 2006, A\&A, 460, 733

Liseau, R. Risacher, C., Brandeker, A., et al. 2008, A\&A, 480, 47

Lissauer, J. J., 1987, Icarus, 69, 249

Liu, M. C. 2004, Science, 305, 1442

Liu, M. C., Matthews, B. C., Williams, J. P., \& Kalas, P. G. 2004, ApJ, 608, 526 Malmberg, D., de Angeli, F., Davies, M. B., et al. 2007, MNRAS, 378, 1207

Mathis, J. S., Mezger, P. G., \& Panagia, N. 1983, A\&A, 128, 212

Matthews, B. C., Kalas, P. G., \& Wyatt, M. C. 2007, ApJ, 663, 1103

Monet, D. G., Levine, S. E., Canzian, B., et al. 2003, AJ, 125, 984

Montes, D., López-Santiago, J., Gálvez, M. C., et al. 2001, MNRAS, 328, 45

Morbidelli, A., Levison, H. F., Tsiganis, K., \& Gomes, R. 2005, Nature, 435, 462 
Najita, J., \& Williams, J. P. 2005, ApJ, 635, 625

Natta, A., Grinin, V., \& Mannings, V. 2000, Protostars and Planets IV (BookTucson: University of Arizona Press), 559

Plavchan, P., Jura, M., \& Lipscy, S. J. 2005, ApJ, 631, 1161

Plavchan, P., Werner, M. W., Chen, C. H., et al. 2009, ApJ, 698, 1068

Reche, R., Beust, H., Augereau, J.-C., \& Absil, O. 2008, A\&A, 480, 551

Rieke, G. H., Su, K. Y. L., Stansberry, J. A., et al. 2005, ApJ, 620, 1010

Schmitt J. H. M. M., \& Liefke C. 2004, A\&A, 417, 651

Siess L., Dufour E., \& Forestini M. 2000, A\&A, 358, 593

Silverstone, M. D. 2000, Ph.D. Thesis, UCLA

Skrutskie, M. F., Cutri, R. M., Stiening, R., et al. 2006, AJ, 131, 1163

Smith B. A., \& Terrile, R. J., 1984, Science, 226, 1421

Smith, P. S., Hines, D. C., Low, F. J., et al. 2006, ApJ, 644, L125

Smith, R., Wyatt, M. C., \& Dent, W. R. F. 2008, A\&A, 485, 897

Spangler, C., Sargent, A. I., Silverstone, M. D., Becklin, E. E., \& Zuckerman, B. 2001, ApJ, 555, 932
Su, K. Y. L., Rieke, G. H., Stapelfeldt, K. R., et al. 2006, ApJ, 653, 675 Su, K. Y. L., Rieke, G. H., Stapelfeldt, K. R., et al. 2008, ApJ, 679, 125 Tacconi, L. J., Genzel, R., Smail, I., et al. 2008, ApJ, 680, 246

Trilling, D. E., Bryden, G., Beichman, C. A., et al. 2008, ApJ, 674, 1086

Tsiganis, K., Gomes, R., Morbidelli, A., \& Levison, H. F. 2005, Nature, 435, 459

Vorobyov, E. I., \& Basu, S. 2008, ApJ, 676, L139

Voss, H., Bertoldi, F., Carilli, C., et al. 2006, A\&A, 448, 823

Wilner, D. J., Holman, M. J., Kuchner, M. J., \& Ho, P. T. P. 2002, ApJ, 569, L115

Wyatt, M. C. 2003, ApJ, 598, 1321

Wyatt, M. C. 2006, ApJ, 639, 1153

Wyatt, M. C. 2008, ARA\&A, 46, 339

Wyatt, M. C., \& Dent, W. F. R. 2002, MNRAS, 334, 589

Wyatt, M. C., Dent, W. F. R., \& Greaves, J. S. 2003, MNRAS, 342, 876

Zuckerman, B. 2001, ARA\&A, 39, 549

Zuckerman, B., \& Inseok Song 2004a, ARA\&A, 42, 685

Zuckerman, B., \& Inseok Song 2004b, ApJ, 613, L65 